\title{
A review of various modalities in breast imaging: technical aspects and clinical outcomes
}

\author{
Sepideh Iranmakani ${ }^{1}$, Tohid Mortezazadeh², Fakhrossadat Sajadian ${ }^{1}$, Mona Fazel Ghaziani ${ }^{1}$, Ali Ghafari ${ }^{1}$, \\ Davood Khezerloo ${ }^{1 *}$ and Ahmed Eleojo Musa ${ }^{3}$
}

\begin{abstract}
Background: Nowadays, breast cancer is the second cause of death after cardiovascular diseases. In general, about one out of eight women (about 12\%) suffer from this disease during their life in the USA and European countries. If breast cancer is detected at an early stage, its survival rate will be very high. Several methods have been introduced to diagnose breast cancer with their clinical advantages and disadvantages.

Main text: In this review, various methods of breast imaging have been introduced. Furthermore, the sensitivity and specificity of each of these methods have been investigated. For each of the imaging methods, articles that were relevant to the past 10 years were selected through electronic search engines, and then the most relevant papers were selected. Finally, about 40 articles were studied and their results were categorized and presented in the form of a report as follows. Various breast cancer imaging techniques were extracted as follows:

mammography, contrast-enhanced mammography, digital tomosynthesis, sonography, sonoelastography, magnetic resonance imaging, magnetic elastography, diffusion-weighted imaging, magnetic spectroscopy, nuclear medicine, optical imaging, and microwave imaging.

Conclusion: The choice of these methods depends on the patient's state and stage, the age of the individual and the density of the breast tissue. Hybrid imaging techniques appear to be an acceptable way to improve detection of breast cancer. This review article can be useful in choosing the right method for imaging in people suspected of breast cancer.
\end{abstract}

Keywords: Breast cancer, Mammography, Sonography, MRI, Nuclear medicine, Optical imaging, Microwave imaging

\section{Background}

The spread of breast cancer has become one of the health challenges in human societies. Breast cancer is the most common type of malignancy in women, and one of the three most common cancers worldwide, along with lung and colon cancer $[1,2]$. In 2012, there were $\sim 1.7$ million new cases of cancer worldwide, and about $31 \%$ of them led to death [3]. Breast cancer is the second leading cause of death after cardiovascular diseases [4]. About one out

\footnotetext{
* Correspondence: d.khezerloo@gmail.com

'Department of Radiology, Paramedical School, Tabriz University of Medical Sciences, Tabriz, Iran

Full list of author information is available at the end of the article
}

of eight women (about 12\%) suffer from this disease during their life in the USA and European countries [5]. The overall prevalence rate and mortality rate has increased in developing countries [3]. However, mortality of breast cancer in North America and the European Union (EU) has decreased, and this is mostly attributable to early detection and efficient systemic therapies $[1,6]$.

There is no clear reason for the occurrence of breast cancer, but some factors increase the risk of cancer such as smoking, obesity, lack of physical activity, alcohol, infections as well as molecular and genetic mechanisms. Clinical studies show that early detection methods increase survival rates up to about 5 years [4]. 
Breast tissue swelling, fixed masses with irregular borders, skin changes such as thickening, discoloration, dimpling, nipple discharge, and breast or nipple pain are important signs of this cancer [7]. Early detection of breast cancer plays an important role in the treatment and control of the disease. If breast cancer is diagnosed early, it has a very high survival rate. To this end, countries have developed some prevention programs. There are currently 3 clinical breast imaging modalities, although manual examination is used as the primary diagnostic tool. The current standard screening and diagnosis method is mammography imaging, which uses low energy 20-30 keV X-rays. According to studies, the sensitivity (true positive) of this method is around $75 \%$, but in middle-aged people whose breast tissues often have a higher mass density, the sensitivity is reduced to about 50\% [8]. So, in some cases, distinguishing between malignant and benign is more complicated [9]. The second method is magnetic resonance imaging (MRI). This method is highly sensitive to cancer detection but has the potential to detect false positives. In other words, its specificity (true negative) is low. Dynamic contrastenhanced MRI (DCE MRI), which uses injectable gadolinium, has been recognized as the most powerful method for detecting breast cancers. The disadvantages of using MRI as a standard breast imaging method are its high cost and time of scanning. However, in high-risk cases, this method is recommended [10]. The third method is ultrasound imaging. The diagnostic power in this procedure strongly depends on the scanner's proficiency and correct selection of ultrasound parameters. On the other hand, in conventional ultrasound procedures, the distinction between cysts and solid tumors is difficult. Hence, Doppler and power Doppler method is used to diagnose breast cancer. Currently, MRI and ultrasound are only auxiliaries to mammography. A study showed that when using mammography and ultrasound simultaneously, the sensitivity of the test for diagnosis was $97 \%$, whereas for mammography with manual examination, the sensitivity was $74 \%[11,12]$. There are other breast diagnostic methods that have received less attention due to some of their challenges and complexities, including tomosynthesis, elastography, photoacoustics, and optical imaging. In this study, we tried to evaluate the performance of conventional breast imaging methods for early detection of breast cancer in women and their effects on reducing mortality rate. Articles that were published with keywords related to breast cancer detection methods were extracted through Pubmed, Scopus, Elsevier, and Google Scholar. In the first step, the keyword "Breast Cancer" with each of the keywords "Nuclear Imaging," "Mammography," "Ultrasound," "MRI," "Optical imaging," and "Tomosynthesis" were searched. For each of the imaging modalities, the articles related to the past 10 years were selected. The titles and abstracts of the articles were reviewed and the most relevant articles were filtered, and finally 8 articles were selected for each imaging method. Overall, about 30 articles were studied and their results were categorized and presented in the form of a report as follows. There are about 20 different imaging methods in the diagnosis of breast cancer that could help to improve the diagnostic of breast cancer. The name and the source of radiations of modalities are summarized in Fig. 1.

Most diagnostic methods of breast imaging are used to diagnose tumors because of the specific anatomy and soft tissue of the breast. In this study, the mechanisms of each imaging technique are described, and then their clinical performances were examined separately below.

\section{Technique}

Mammography

A mammogram is a two-dimensional image that helps to identifying morphologically suspicious findings in breast cancer. These findings include masses, asymmetric calcifications, and deformed breast areas. In this method, the breast tissue is pressed by a plate, and then $2 \mathrm{D}$ radiographic images are produced by penetrating low-energy $(20-32 \mathrm{kVp}) \mathrm{X}$-rays through the tissues. A standard screening mammogram is obtained in the oblique views (MLO) and craniocaudal (CC) of each breast (Fig. 2). If the lesion is suspected, other imaging views including point compression, magnification, and actual lateral views are required to determine local features and abnormalities. Breast Imaging Reporting and Data System (BIRADS) by the American College of Radiology standardizes mammography terminology [13].

Since the introduction of mammography about 30 years ago, breast imaging has improved significantly with this method. Film-screening mammography is the gold standard in breast cancer imaging. However, it is restricted to detection in dense breast tissues [14]. Initial studies on the clinical function of mammography have shown that this method reduces the mortality rate about $20-40 \%$ [15]. Mammography, on the other hand, has high false positives due to the overlap of normal fibroglandular tissues in 2D imaging and the appearance of abnormalities resembling cancer and further inducing unnecessary biopsies [15]. The benefits of mammography do not always weigh on its disadvantages. One of the major disadvantages of mammography is inducing radiation dose in a high sensitive tissue such as breast. Studies have shown that a complete mammography imaging induces approximately 1-3 mGy dose into the breast tissue itself, which can increase the risk of cancer in the individual [16]. The sensitivity of mammography has an inverse relationship with breast density [14]. High-density breast implies more fibroglandular tissue 


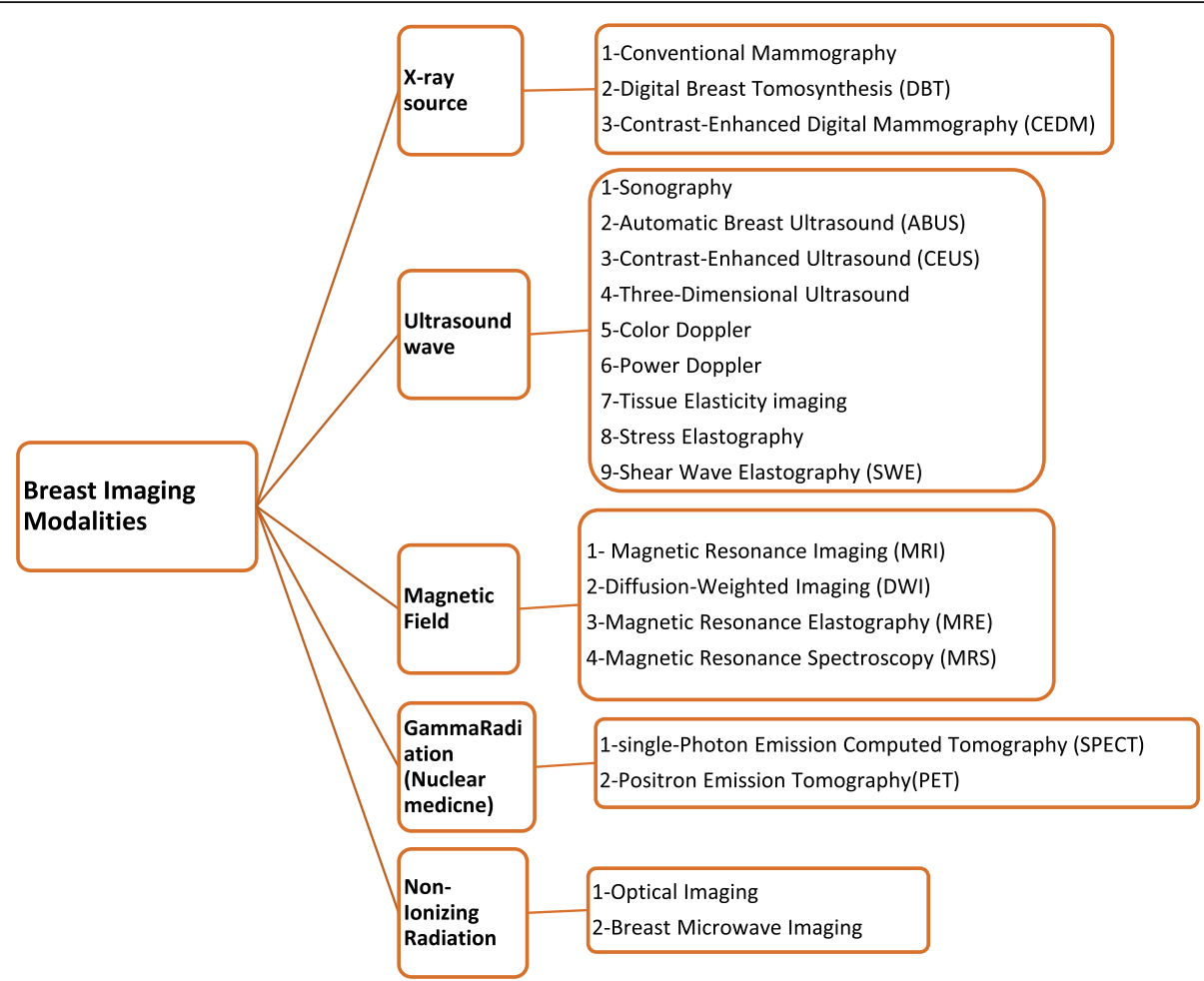

Fig. 1 Different imaging methods in the diagnosis of breast cancer
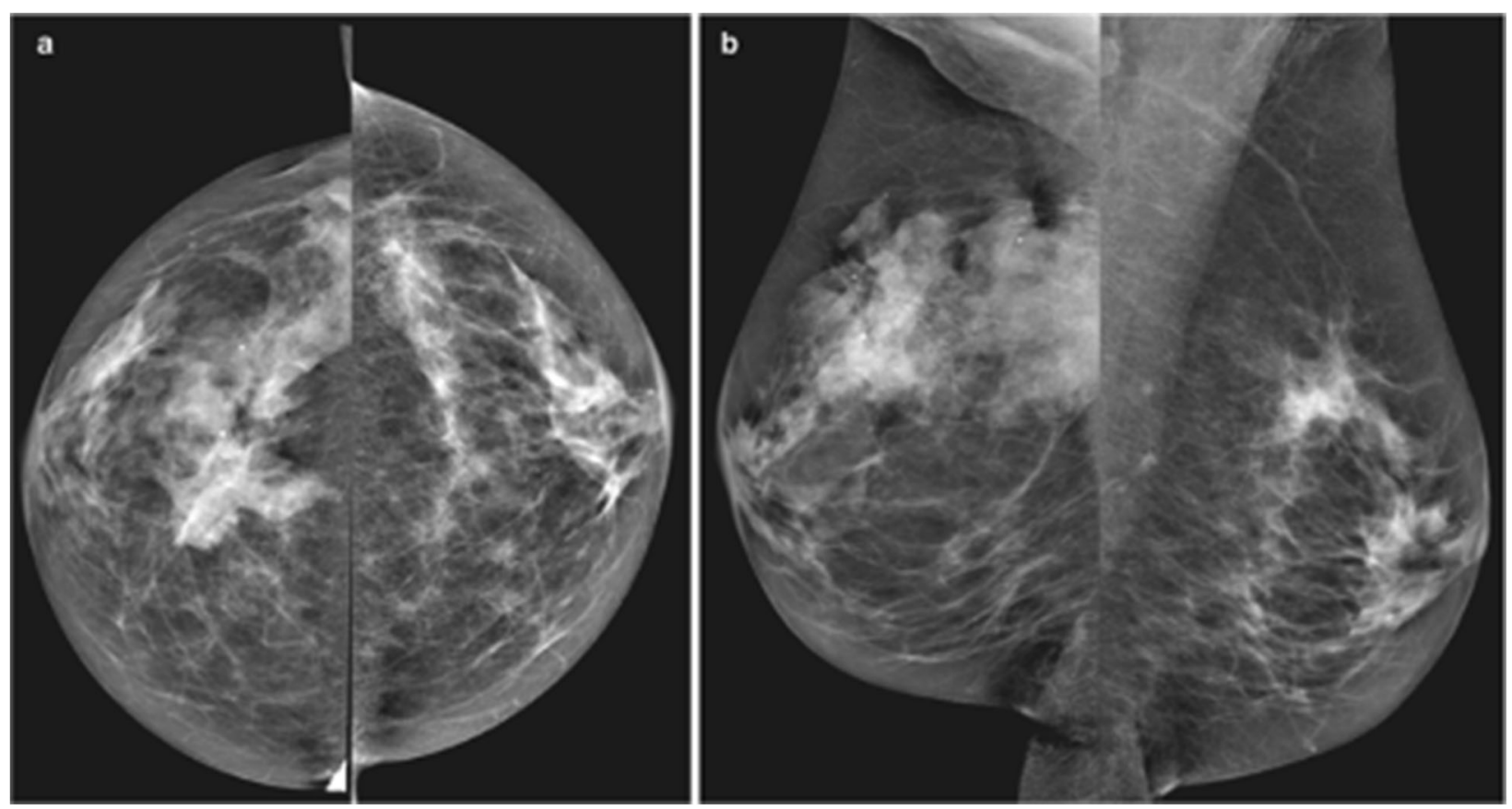

Fig. 2 Mammogram of ductal carcinoma in a 72-year-old woman. a CC view. b MLO view [13] 
and less adipose tissue. The sensitivity of mammography in the breast tissue of 50-year-old women varies from 68 to $90 \%$ and in women aged $40-49$ is about $62 \%$. The specificity of mammography ranges from 82 to $97 \%$ [8]. According to the results of Breast Cancer Surveillance Consortium (BCSC), sensitivity of mammography is about $57 \%$ in women with dense breasts and in women with high adipose tissue sensitivity increases up to $93 \%$ [17]. Concerns about the side effects of ionizing radiation used in mammography are still present, and some studies have shown that mammography may increase the incidence of breast cancer. The United States Preventive Services Task Force (USPSTF) has recommended mammography every 2 years and after the age of 50 . Heterogeneous and dense breast parenchyma in digital mammography shows better sensitivity than film-screen mammography, but in general, both methods are less sensitive in dense breasts [18]. The introduction of digital mammography has had many benefits including easy storage, retrieval and transfer of images, and better detection of dense breast issue.

Other studies have reported different results on mammography function. After 17 years of follow-up in the UK, it has been shown that mammography screening, starting at age 39-41, has no benefit in reducing cancerrelated mortality. Two randomized Canadian study found no significant reduction in the risk of death from breast cancer with mammographic screening compared to screening using breast clinical trials. On the other hand, mammography detects invasive lobular and in situ carcinomas, which includes 8 and 14 percent of all cancers difficultly [9].

Digital mammography has advanced to newer digital tomosynthetic technologies and contrast-enhanced digital mammography [14].

\section{Digital breast tomosynthesis (DBT)}

This method is a subset of the mammography procedure, with the difference that the $\mathrm{X}$-ray tube rotates around a narrow angular angle $\left(15-60^{\circ}\right)$ from the compressed breast tissue and produces 3D breast information [14]. DBT images are generated from repeated exposure to the breast tissue at various angles and reconstructed as half-millimeter slices (Fig. 3). Various studies have shown that this method increases the patient's radiation dose by $20 \%$, but the cancer detection rate increases about $15-30 \%$ and the recall rate decrease about $15-20 \%$ [13]. The main advantage of tomosynthesis is the detection of masses and lesions that may not be seen in conventional mammography due to overlap with dense breast tissue. The sensitivity of tomosynthesis is high and false-positive detections are decreased. Hence, the staging of breast cancer will be more accurate using this method. It is better to detect non-calcified lesions with DBT although classification lesions also have similar or better results in DBT than conventional mammography. In the USA, combining digital breast tomosynthesis with digital mammography has reduced the recurrence rate by up to $30 \%$ [14]. However, the radiation dose in DBT is $8 \%$ higher than the standard

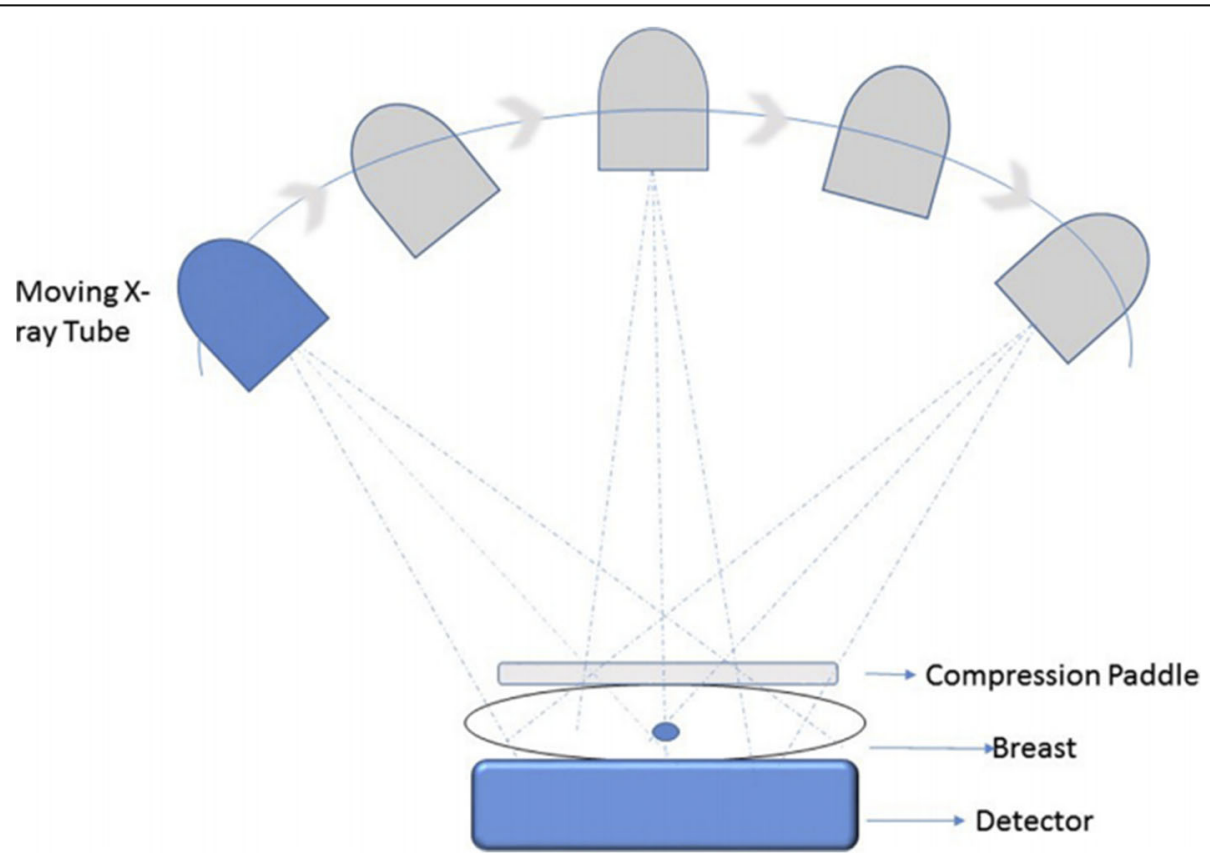

Fig. 3 Breast digital tomosynthesis [15] 
digital mammography. In conclusion, low sensitivity in the detection of microcalcifications is the major limitation of this technique [18]. DBT causes a $27 \%$ increase in the diagnosis of breast cancer and a $15 \%$ decrease in false-positive findings [15]. In general, DBT is better than mammography in diagnosis and screening. On the other hand, it reduces the patient's recall for other medical examinations [15].

\section{Contrast-enhanced digital mammography (CEDM)}

The clinical significance of the angiogenesis pattern in breast cancer screening is one of the important diagnostic tools, as studies have shown that the intra-tumor microenvironment is highly correlated with the likelihood of metastasis [19]. Contrast-enhanced mammography represents the angiogenic pattern of the masses, which depicts anatomical information of the tissue. The sensitivity of this procedure is high, about $85-90 \%$, but it has a low specificity compared to conventional mammography and also has limitations in the diagnosis of ductal carcinoma of the breast. Another limitation of this method is the high cost, difficult access and lack of knowledge of the technologist and patient [14]. In general, the CEDM method is two types, both using iodinebased contrast media and digital mammography devices.
In the first method, images of before and after contrast media injection is subtracted (temporal subtraction technique), and the second method is dual-energy or contrast-enhanced spectral mammography (CESM) technique that produces images using X-rays at high and low energies [14]. In the subtraction technique, two images with high-energy X-rays are taken before and after the contrast injection without moving the patient and without altering tissue compression. The beam energy is 45-49 kVp (conventional mammography: 26-32 kVp energy), so the patient dose is similar to conventional mammography and about 1-3 mGy. The whole scanning time in this method takes about 15 min [19]. Images are taken immediately after injection of the contrast media at successive times and observing the temporal pattern of vascular filling and emptying. In this dynamic imaging, one can draw a time-diagram of the vessels being filled with contrast material (Fig. 4) [20].

The dual-energy technique (CESM) produces a picture from material attenuation coefficients to the beam energy. In this method, after contrast material is injected, two images, one at high energy and the other immediately after (with a time interval of less than $30 \mathrm{~s}$ ), are taken at low energy from the breast tissue and the total imaging time takes between 5 and $10 \mathrm{~min}$. Afterward,

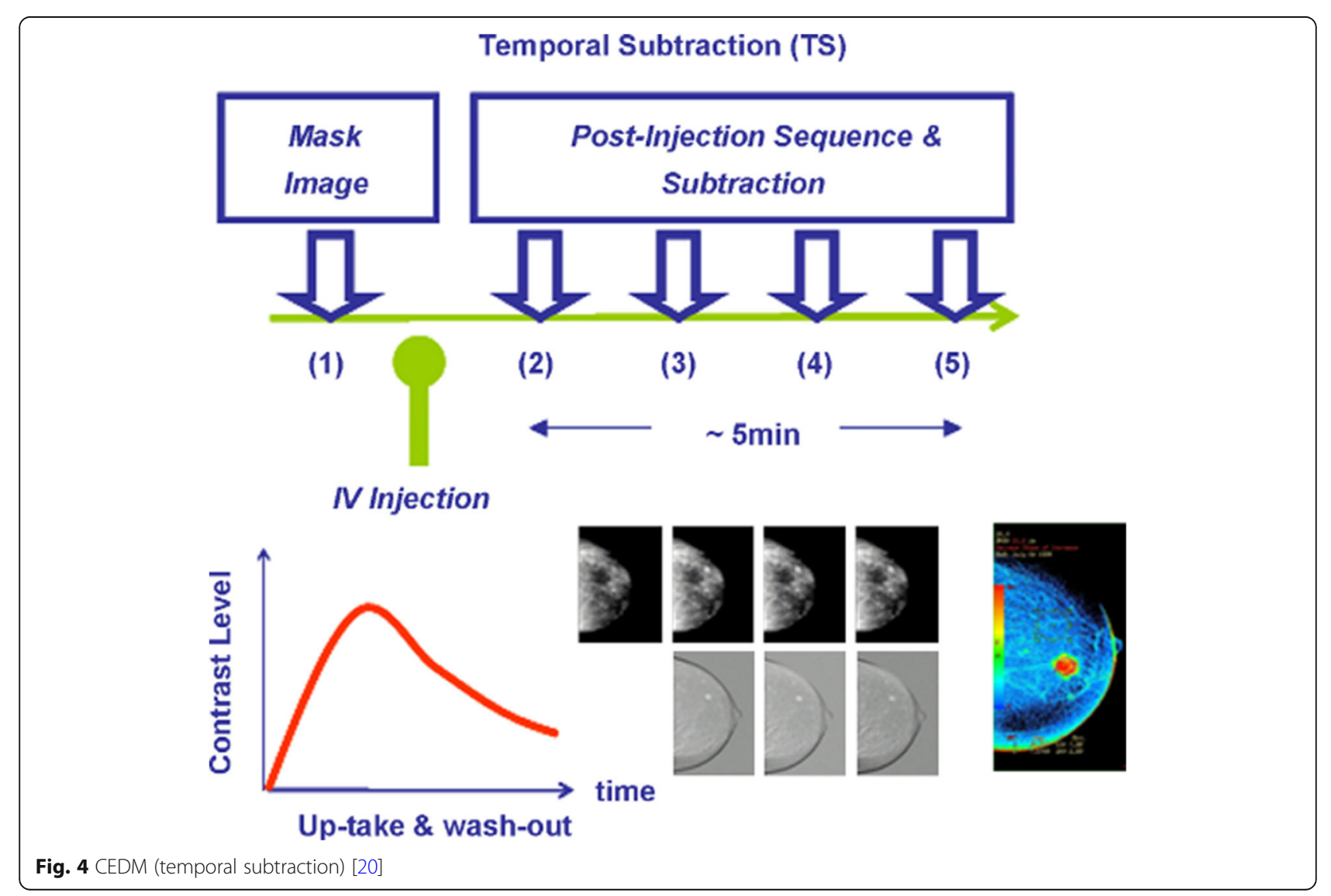


these two images are combined in order to improve the image contrast. Since the magnitude of the produced signal in the receiver detectors is proportional to the energy of the received photons, the energy threshold can be applied to the detectors. The information of two images is useful for differentiating different parts of the breast tissue such as the cystic masses from the tumor (Fig. 5) [14]. In fact, dual-energy imaging is a new technology in digital mammography that aims to reduce the average breast dose and also reduce $98 \%$ of the radiation dose caused by scattered rays [18]. Preliminary results comparing the dual-energy technique with conventional mammography, ultrasound imaging, and breast MRI show a better diagnosis of suspected lesions with this method to previous ones; however, the detection of hypervascular breast lesions using this technique is poorer than breast MRI [21]. The sensitivity of this method (about 93\%) has increased compared to mammography while its specificity is $63 \%$ [22]. The dose achieved in this procedure is about $20-50 \%$ higher than the usual mammographic dose, depending on the size of the patient's breast tissue. The dual-energy method does not produce temporal and dynamic information but it can produce images from several projections; therefore, the time of imaging and motion artifact is low and the condition is more feasible to the patients [20].

Both contrast-enhanced mammography (CEDM) techniques are commonly used to evaluate tumor angiogenesis in specific individuals, but despite their high sensitivity, they have low specificity and have limitations in the diagnosis of breast ductal carcinoma. The main disadvantages of this method are the high cost, difficult access and lack of familiarity of the technologist, and the patient with this method. The initial clinical results show that CEDM has higher diagnostic accuracy than mammography alone or combining mammography with ultrasound. Adding differential CEDM to conventional mammography enhances the quality of diagnosis and sensitivity. Combining tomosynthesis with CEDM reduces the impact of surrounding soft tissues and increases the contrast between the tumor and surrounding tissues [14]. Thus, its sensitivity increases by about $35-59 \%$ even for dense parenchymal breasts [23] Fig 6.

\section{Sonography}

In this method, the ultrasound wave is transmitted to the tissue by a probe at frequencies ranging between 3 and $12 \mathrm{MHz}$. The different organs reflect the ultrasound to the probe because of differences in their acoustic impedance. The magnitude of reflected wave intensity produces a gray pattern on the screen [24]. Advancements in transducer design, electronics, computers, and signal processing have improved the quality of ultrasound images for breast cancer detection. Ultrasound along with mammography is used to determine the nature (benign or malignant) of solid masses. Also, Doppler ultrasound

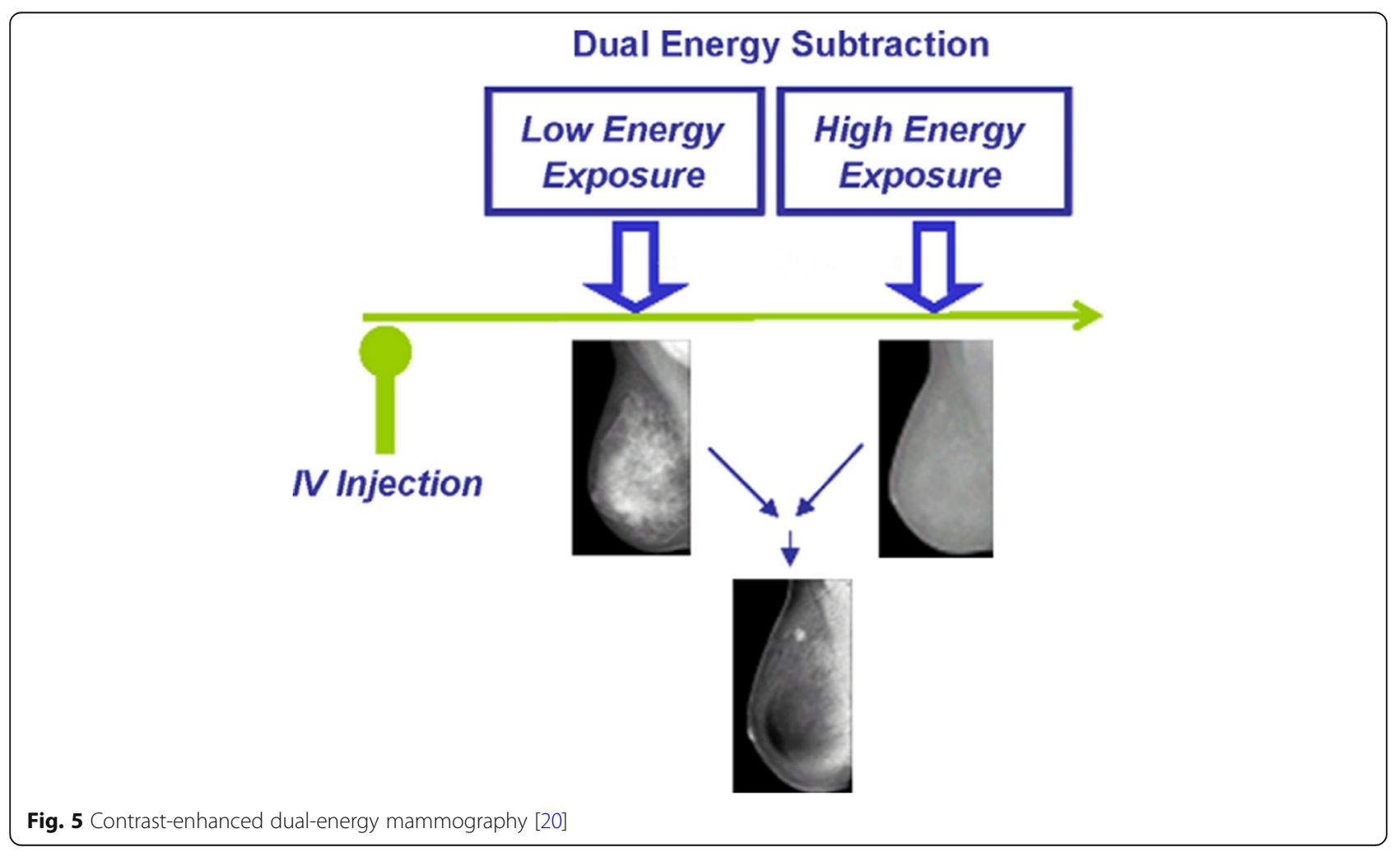



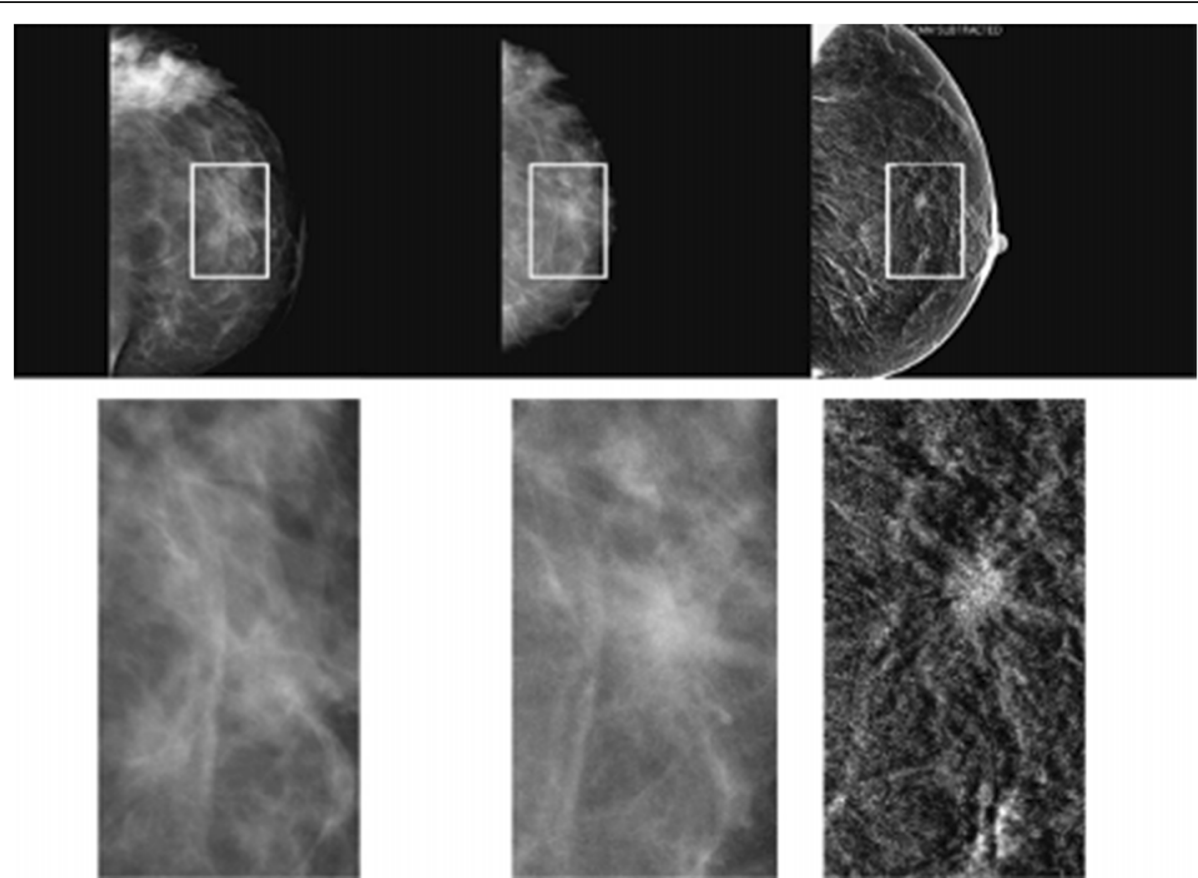

Fig. 6 Conventional mammogram (left and middle). Contrast-enhanced dual energy (right) [23]

and contrast media are used to measure tumor blood flow and tumor vascularization. The convenience and ability to produce simultaneous images is why it is used to guide breast biopsies and other interventional processes. B-mode (grayscale) is one of the most common ultrasound techniques. Ultrasound can evaluate the morphology, orientation, internal structures, and margin of lesions in dense breasts on several plates. Evaluation of these features helps to differentiate benign and solid breast lesions [25]. Sensitivity increases to $97.3 \%$ and specificity to $76.1 \%$ by adding ultrasound imaging to conventional breast cancer screening methods (mammography and physical examination) [26]. Various advances have been made in ultrasound technology, including 3D ultrasound, color Doppler, power Doppler, automated breast ultrasound (ABUS), and sonoelastography. 3D ultrasound and ABUS collect volumetric information from the whole breast.

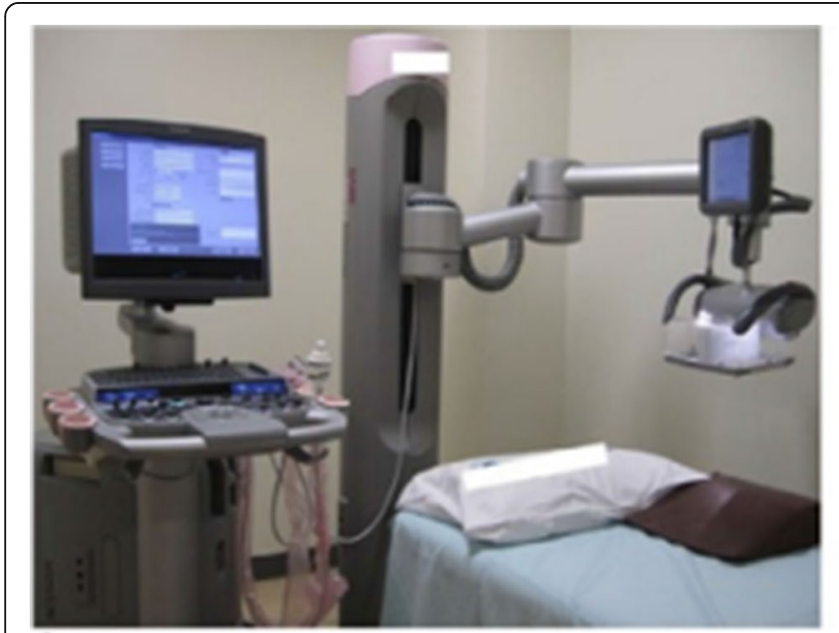

A breast and an automated scan is performed to obtain a series of 2D images. Depending on breast size, more than three scans per breast may be required [25]

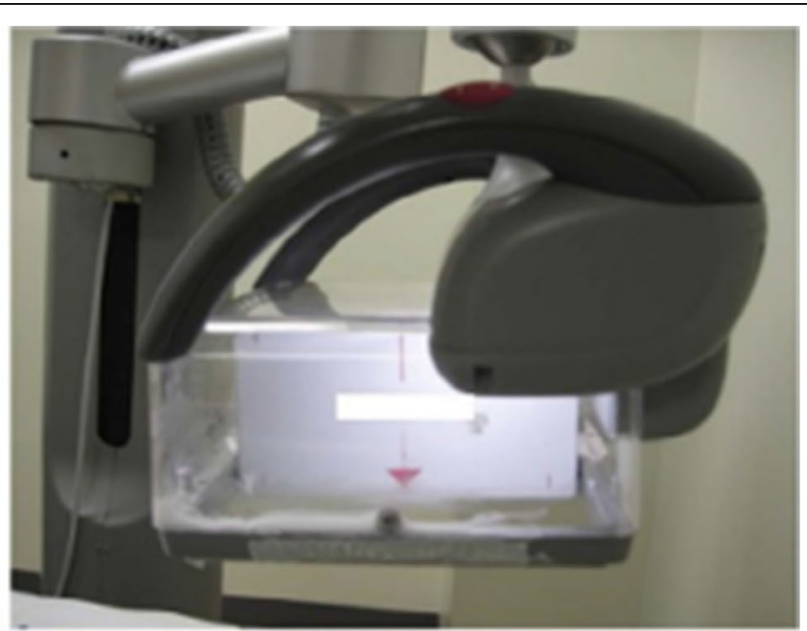

B 


\section{Automatic breast ultrasound (ABUS)}

The advantage of ABUS is its high diagnostic accuracy and better determination of lesion size [14]. From each breast, three positions are obtained by the operator with flat ultrasonic plates. The images are then reconstructed in 3D. This technique is a good method for women with dense breasts. It can reduce patient recall rates (less than 2.5\%) [13] (Fig. 7). The sensitivity of ABUS in the diagnosis of breast cancer is $79 \%$ and its specificity is $83.3 \%$ [25].

\section{Contrast-enhanced ultrasound (CEUS)}

Tumor vascularization depends on the size and severity of its pathology. There are disorders of vascular distribution in malignant breast lesions. CEUS is used in clinical research to observe different vascular structures. CEUS uses vein injection of gas microbubbles to improve the return of the waves. Microbubbles are certain gasses that are trapped in different types of capsular shells. Their diameters range from 1 to $7 \mu \mathrm{m}$. Microbubbles are more echogenic than red blood cells and do not pass through the vascular wall. Due to the differences in acoustic impedance and compression between the microbubbles and surrounding tissues, the ultrasound contrast media mainly acts as a nonlinear impurity. Non-linear imaging techniques, including pulse reversed harmonic imaging, power Doppler, and subharmonic imaging, are used to reduce bubble destruction and provide better images. The combination of micro-bubbles with non-linear imaging techniques can show vascular morphology. After the injection of contrast media, different phases of perfusion, initial phase (0-1 min), intermediate phase (1-4 $\mathrm{min}$ ), and delay phase (4-6 min) are obtained. Investigation and analysis of these phases in different lesions allows differentiation of lesions. The sensitivity and specificity of this method for diagnosis of breast lesions are $100 \%$ and $87.5 \%$, respectively [25].

\section{Three-dimensional ultrasound}

There are two main types of 3D ultrasound. The first type is the use of two-dimensional imaging equipment with special mechanics, which finally reconstructs the 3D ultrasound volume. The second uses a matrix array converter that electronically scans a $3 \mathrm{D}$ volume. The matrix array creates a beam in both positions and forms a pyramidal volume [25].

\section{Color Doppler}

A conventional ultrasound technique is helpful in diagnosing tumor angiogenesis [14]. The color Doppler estimates the local blood flow using mean Doppler shift coding in the region of interest and colored it. It is optional to choose the mean frequency shift as a parameter to display blood flow in color Doppler. Malignant breast lesions usually increase the Doppler signal. Color Doppler imaging is highly dependent on technical factors. To optimize vascular flow sensitivity, consider scale reduction by using power Doppler imaging (removing flow direction) and increasing color rate. Color Doppler sensitivity increases with color box size reduction (Fig. 8). Another influential factor in color Doppler is the reduction of the transducer pressure on the breast. As the breast lesions are relatively superficial, the vessels can be easily closed when squeezed between the transducer and chest wall [27].

\section{Power Doppler}

It is a technique that encodes pulse power into the Doppler signal and displays it in a single color. This parameter is different from the frequency shift.

Frequency is determined by the speed of red blood cells (RBCs). So the signal strength depends on the amount of blood present in the target area.

The flow direction in this method is usually not important. The advantages of power Doppler over color Doppler are its high sensitivity to blood flow, better resolution especially in edges and delineation of blood flow continuity. Power Doppler is widely used in the diagnosis of solid breast lesions [27].

\section{Tissue elasticity imaging (sonoelastography)}

In recent decades, many efforts have been made to improve the sensitivity and specificity of breast cancer diagnosis in ultrasound, including the use of ultrasound for imaging tumor elasticity. Solid tumors are physically more rigid than their surroundings. The stiffness of the tissue is determined by Young's modulus (also known as elasticity, which is the deformation of tissue due to pressure). The soft tissue elasticity ranges from 1 to $100 \mathrm{kPa}$. Fibroadenomas are two times stiffer than normal breast parenchyma and elasticity of breast cancers is about 15 times stiffer than soft tissue. This procedure produces sonograms of the tissue before and after compression. The solid masses are less deformed and appear darker in elastogram. Malignant masses are also solid masses that will look darker than benign masses.

Benign masses can be either lighter or darker than the surrounding environment. A comparison of the size of the breast lesions in elastogram and B-mode images reveals the differences between benign and malignant lesions. Cysts and fibroadenomas have the same sizes in both types of elastogram and B-mode images, but carcinoma is seen larger in elastograms (Fig. 9). Sonoelastography has a higher specificity than B-mode ultrasound [14]. The sensitivity and specificity of sonoelastography are $88.5 \%$ and $92.7 \%$, respectively [29]. The distinction between benign and malignant tissues in sonoelastography is important and is still subject of discussion [28]. In general, the sonoelastography technique is performed in two ways: stress elastography (SE) and shear wave 


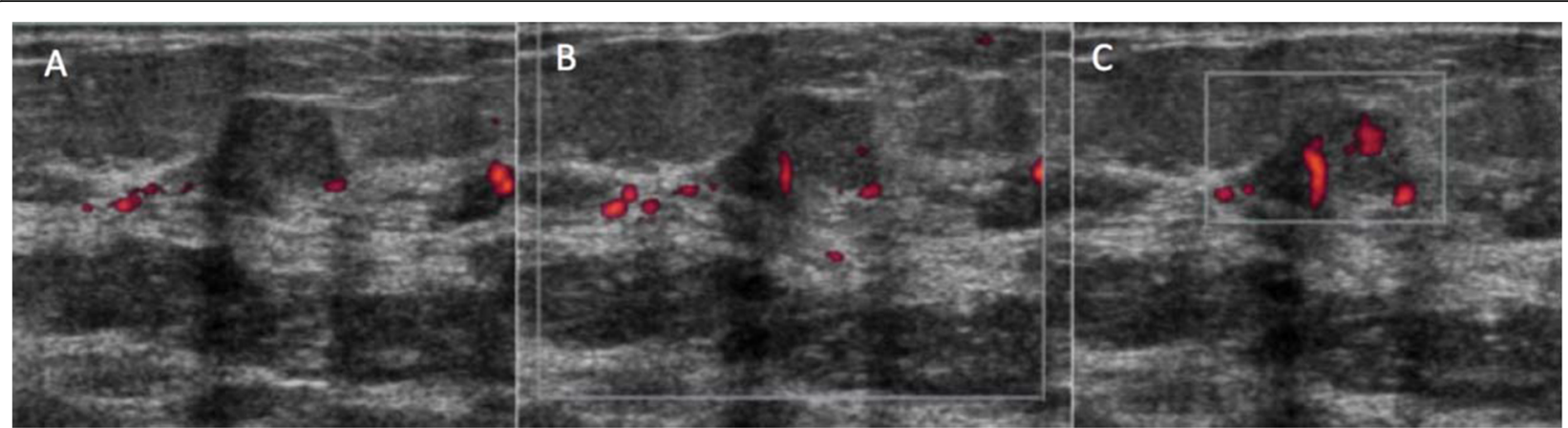

Fig. 8 Color Doppler imaging. a, c Decreasing the Doppler box size, improves the sensitivity to flow [27]

elastography (SWE) [30]. Studies have shown that there is no significant difference between SE and SWE ultrasonography, and both have similar sensitivity and specificity when compared with conventional sonography [28].

\section{Stress elastography (SE)}

This method examines how the texture changes when an external force is applied to it. Soft tissue changes more than stiff tissue in the face of an ultrasound wave. A static force is applied to the tissue, and then the desired elastogram image is obtained and combined with the non-force state image (Fig. 10). This pressure can be due to the self-controlled movement of the probe, the patient's motion (breathing or heart movement, or both), or an external factor. If the compression or propagation force is too large, it causes elastogram noise, and if compression is not sufficient, no elastogram is obtained. The sonoelastography technique does not require complex software but is highly dependent on user performance $[28,30]$.

\section{Shear wave elastography (SWE)}

Shear waves are transverse ultrasound waves produced as a result of volume changes due to the ultrasonic pressure in the material. These waves move in stiff tissues like malignant mass faster than soft tissues.

Wave speed is in the range of $1-50 \mathrm{~m} / \mathrm{s}$ and the frequency is $10-2000 \mathrm{~Hz}$. Shear wave velocity is expressed in the unit of $(\mathrm{m} / \mathrm{s})$ or as Young's modulus in kilopascal. In this method, a dynamic compression applied by the ultrasound itself to the tissue in the imaging area then

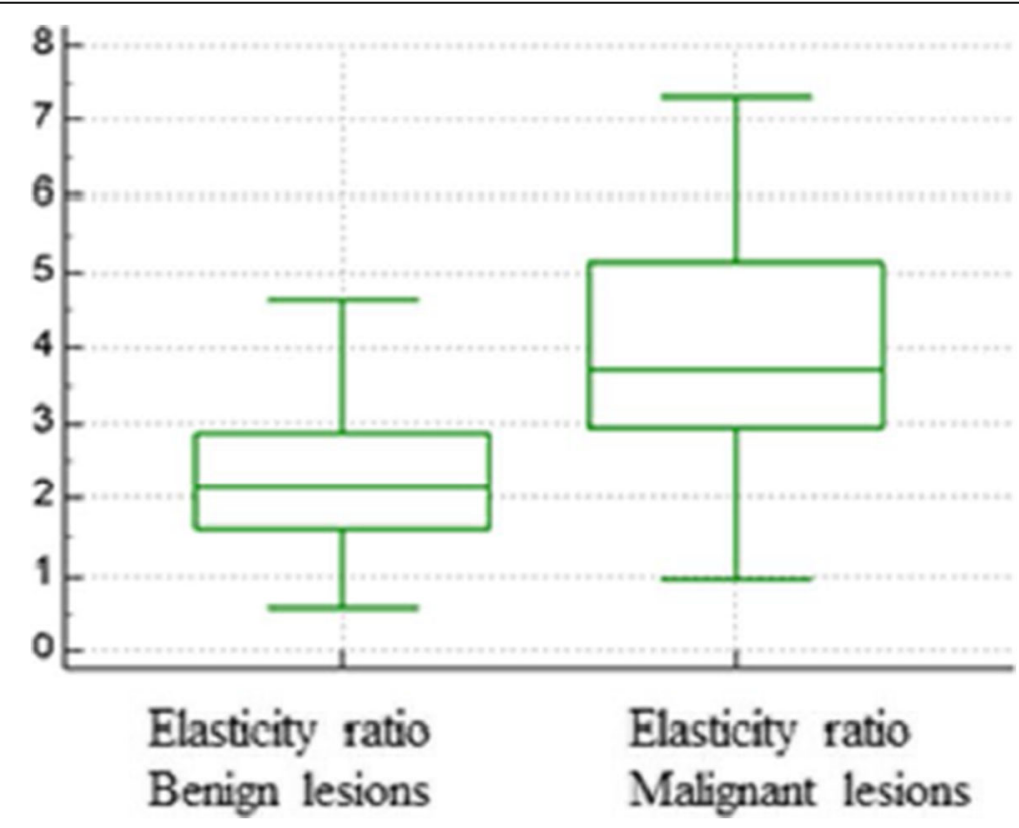

Fig. 9 Elastic ratio of malignant and benign mass [28] 


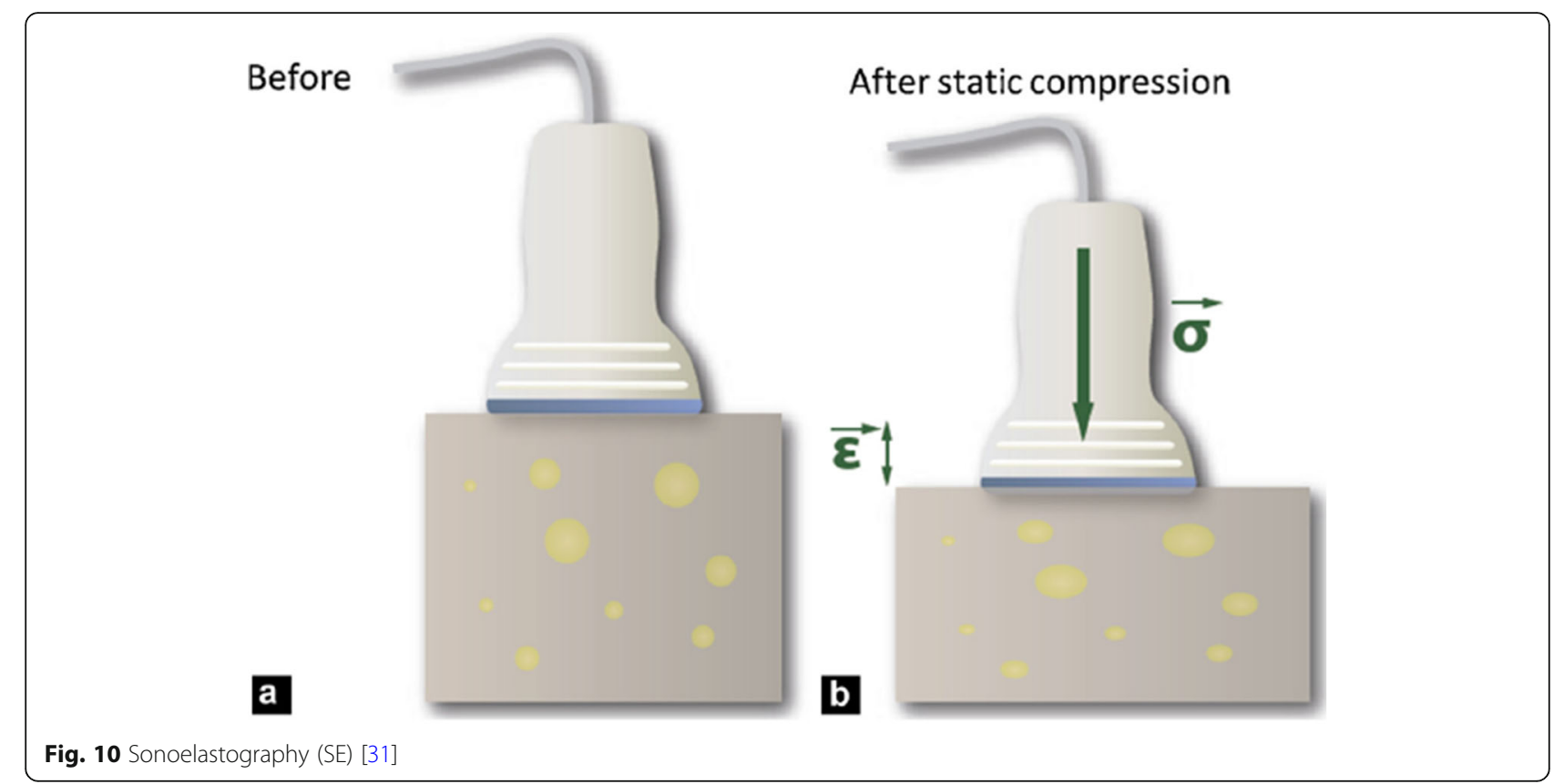

wave velocity is simultaneously manifested and converted to an image. The repeatability of this method is high. When a region (ROI) is selected, pre-compression is removed and two-dimensional SWE is turned on. The patients must remain static during the acquisition of information. Color scales are considered for the lesions observed [28] (Figs. 11 and 12).

\section{Magnetic resonance imaging (MRI)}

Magnetic resonance imaging has become widely used due to the advances in surface coil technology, the introduction of new contrast agents and fast imaging sequences. In this method, the image is produced using the magnetic properties of the hydrogen atoms in the tissues. Although MRI is not commonly used for breast

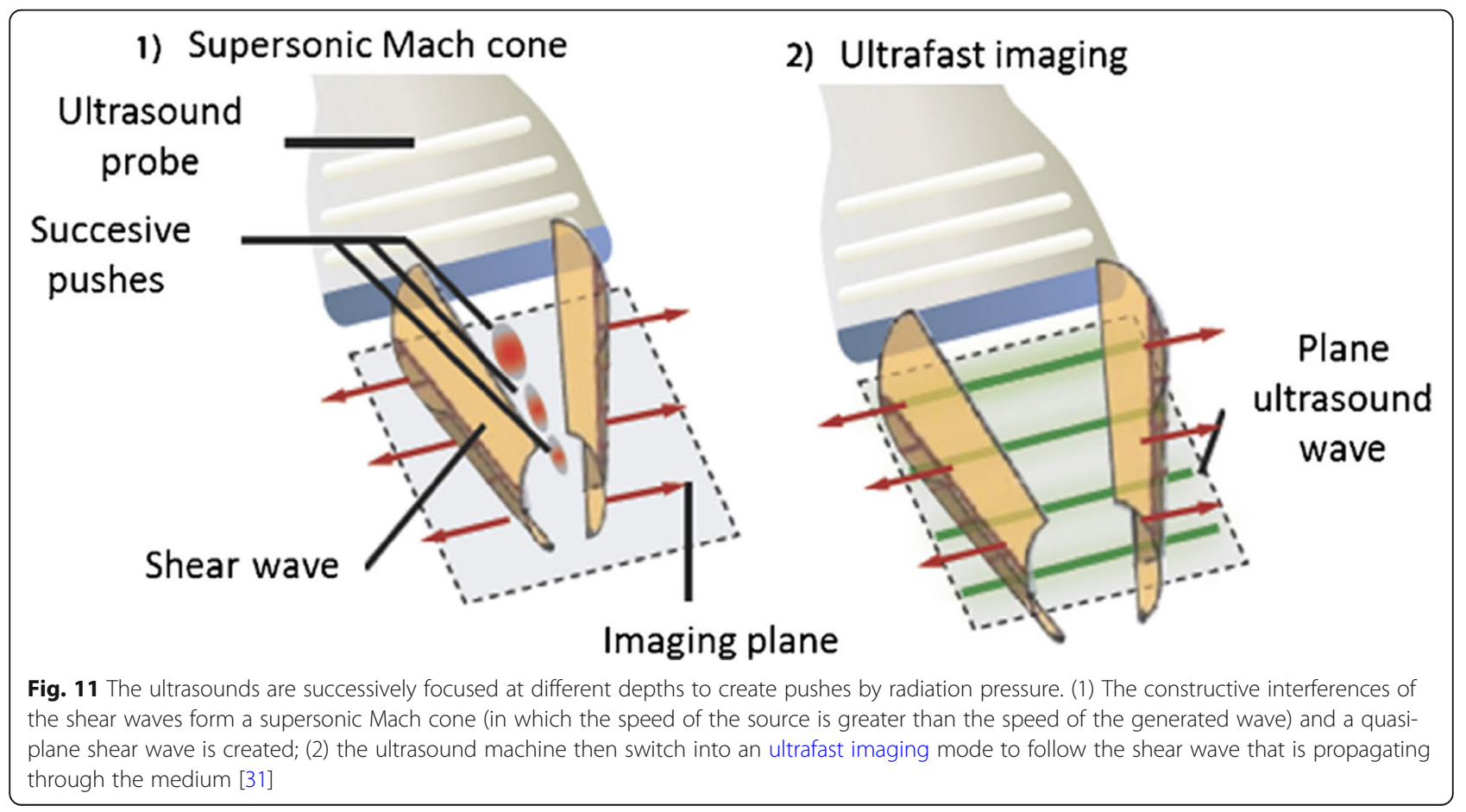



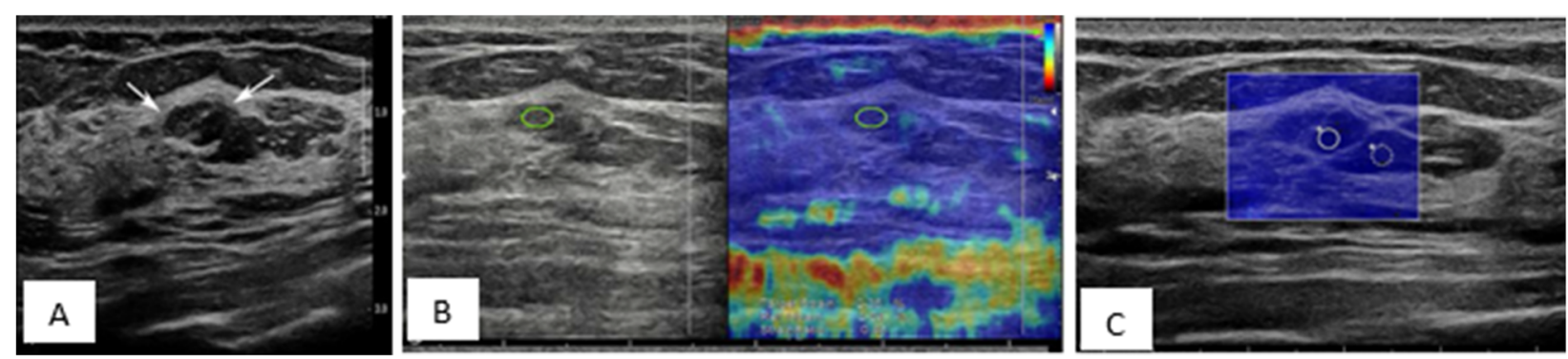

Fig. 12 An abnormality detected via screening mammography in a 51-year-old woman (a). Grayscale ultrasound shows an indistinct oval heterogeneous echoic mass (arrows) (b). SE revealed intra-region heterogeneity with malignancy elasticity index and c SWE obtained two regions with malignant elasticity [28]

cancer imaging, it is a suitable method for screening patients with a high risk of breast cancer (20-25\%) [14]. According to the American Cancer Society (ACS), highrisk patients are recommended to be screened annually using MRI imaging. Additionally, the measure of high risk is those individuals with mutations in the BRCA1 and BRCA2 genes who have a history of chest radiotherapy at ages 10 to 30 years [18]. MRI imaging is more sensitive than mammography and ultrasound in the diagnosis of breast cancer and it is relatively costeffective. Studies have shown that MRI has detected 14.7 new cases of cancer per 1000 people when used as a complementary method in people who have already had mammography and ultrasound [18]. In this method, the contrast material is administered and the patient is lying on a special coil to hang the breast tissue. Different MRI sequences can be utilized and it is recommended that both breasts be imaged simultaneously. In 2017, a metaanalysis compared and analyzed the sensitivity and specificity of MRI imaging techniques with mammography (MG). Results of 11 previous studies showed that MRI and MG sensitivity were $92 \%$ and $75 \%$, respectively, and their specificity was $71 \%$ and $70 \%$, likewise [32]. Ellen Warner, in a cohort study between 1997 and 2004, investigated the sensitivity and specificity of Gd-DTPA contrast-enhanced MRI imaging in high-risk individuals who were induced by BRCA1 and BRCA2 gene expression in 649 patients between the ages of 35-49. Compared with the mammography method, it was found that sensitivity for MRI was $75 \%$, mammography $40 \%$, and MRI with mammography $94 \%$. The specificities are $81 \%$, $93 \%$, and $77 \%$, respectively. The sensitivity of the MRI method for patients with BRCA1 gene expression was better than those with BRCA2 gene expression which was $92 \%$ and $58 \%$, respectively, while the sensitivity was worse for mammography ( $23 \%$ and $50 \%$, respectively). However, when MRI and mammography were used together, the sensitivity of breast cancer for both genes was $92 \%$ [33]. Another major challenge of MRI for breast imaging along with the high cost and time of the scan is patients' difficulties in maintaining proper posture. In a previous study, patients' comments on CEDM and MRI were compared. Seventy-nine percent of patients tended to have CEDM, despite intravenous iodine contrast injection. It should be noted, however, that the radiation dose in the CEDM method is about $20-80 \%$ of the usual mammographic dose. Moreover, the sensitivity and specificity of the two MRI and CEDM methods are similar [34]. Until 2011, five MRI screening studies have been performed, with sensitivity results ranging from 68 to 91\%. Prospective clinical trials, however, have shown that although MRI makes early diagnosis possible, for those at high risk, early diagnosis has little effect on increasing patient survival. Alongside with routine MRI imaging protocols based on $\mathrm{T}_{1}, \mathrm{~T}_{2}$, and proton density providing anatomical images, there are other special MRI techniques that are capable of generating dynamic information of water molecule diffusion (DWI), maps of water molecule diffusion (DTI), modulation of blood cell perfusion (PWI), and MRI-based and biochemical elastography, including magnetic resonance spectroscopy (MRS), which extracts the chemical content of lesions [10]. The following are specific MRI techniques used to diagnose breast cancer.

\section{Diffusion-weighted imaging (DWI)}

In the body environment, the pathway for the release of water molecules is not random due to the presence of cell membranes and other physiological barriers. The diffusion-based imaging method of water diffusion among the internal cellular molecules provides a clear picture and the difference in the pattern of water diffusion can provide contrast to the loss of normal tissue tumors. Studies have shown that the sensitivity and specificity of this method in distinguishing benign from malignant breast tissue lesions are $80 \%$ and $81 \%$, respectively. This method was introduced more than 20 years ago leading to an increase in the diagnostic accuracy of MRI and enabling distinction between benign and malignant breast masses. DWI is a fast technique (120180 s scan time) which does not require intravenous gadolinium injection and is cheaper than other contrast- 
enhanced MRI techniques. DWI is a very sensitive method, even in micrometer-sized structures, based on Brownian molecular motions. The water in the biological tissue produces the image. Most DWI sequences use echoplanar imaging (EPI). EPI is a fast imaging technique for reducing scan time and motion-induced degradation and increases the signal-to-noise ratio. Water permeability in tissue is inversely related to cellularity and cell membrane integrity. Therefore, the signal intensity in DWI is high in regions with limited propagation such as cancerous tissues. Molecules with high T2 relaxation times produce higher signal intensities in DW images [14, 15, 35] Fig. 13.

\section{Magnetic resonance elastography}

Magnetic resonance elastography (MRE) is used for each organ to determine the biomechanical properties of its tissue and changes caused by disease. Adequate transmission of shear waves in the organ of interest is one of the important factors for measuring MRE. This procedure can be difficult for deeper organs such as the pancreas and heart but is easier for the breast due to manual mass examination. In carcinogenesis, biomechanical changes in the tissue are evident. An animal model study showed that Young's modulus (stress-strain ratio) was 15 times greater than that of fibroglandular tissue and surrounding adipose tissues, whereas benign fiber adenomas were only twice as large as the surrounding tissue, which indicates that benign and malignant lesions differ according to their elasticity. Therefore, measuring biomechanical parameters with MRE can provide valuable diagnostic information. The MRE uses a coil as a motor (engine-coil) placed near the breast to produce shear waves. The magnetic fields produced by these coils cause artifacts in the image. To overcome this problem, the coils are placed at a distance from the breast using a tensile-compression system. This improves image quality and is convenient for the patient [36]. Similar to sonoelastography, MRE provides information on tissue stiffness to determine lesion characteristics [14]. The 50-100 $\mathrm{Hz}$ range is the most appropriate frequency range for MRE in the body [36] (Figs. 14, 15, and 16).

\section{Magnetic resonance spectroscopy (MRS)}

Magnetic resonance spectroscopy (MRS) can be performed in conjunction with magnetic resonance imaging (MRI) to obtain information on the chemical content of
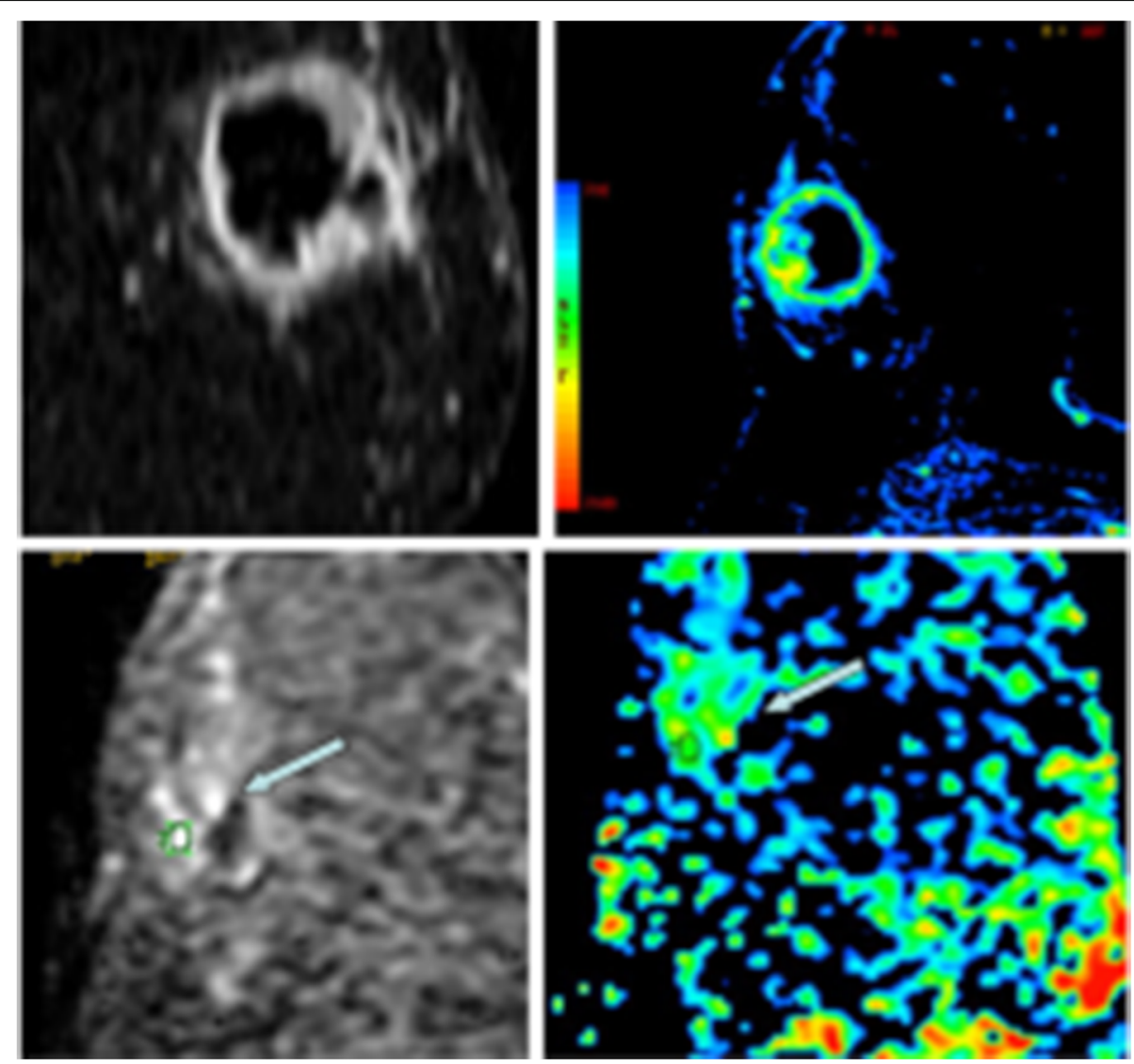

Fig. 13 DW image and a color map of a large breast tumor. Due to the necrosis of the tumor in the ring shown, the diffusion distribution is limited [14] 


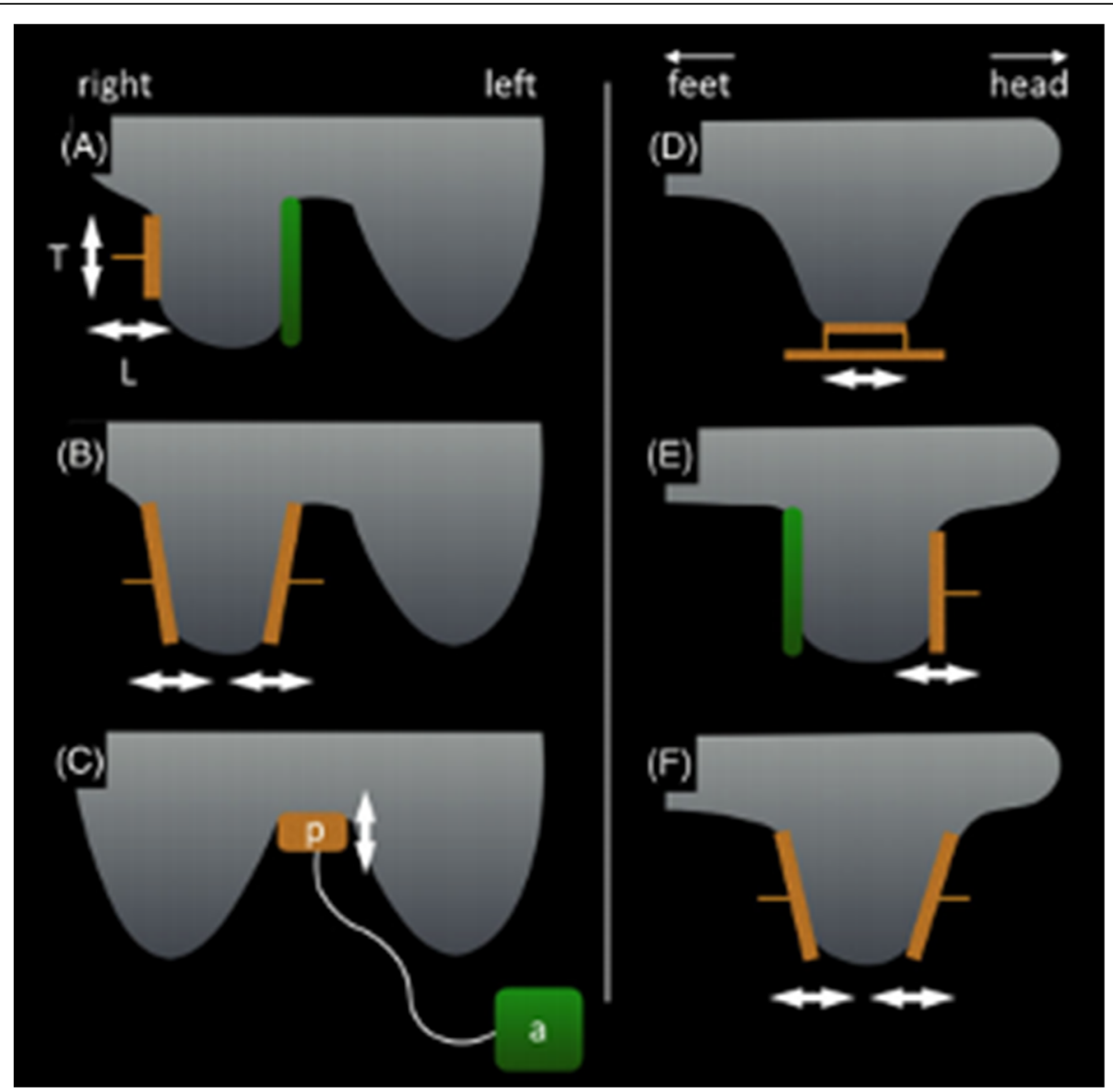

Fig. 14 Simulation map of transmission methods of mechanical waves to the breast tissue. The coil around it is not drawn. a The breast is positioned against a stationary plate in the inner part and mechanical waves are introduced from the outer portion in the longitudinal direction $L$ or transverse $T$. b Two mechanical wave transmitters are positioned obliquely on the inner and outer sides of the breast. c Mechanical waves are transmitted to the sternum by an unknown transmitter. $\mathbf{d}$ A shear plate is placed in front of the breast. $\mathbf{e}$ The breast is fixed at the bottom of the plate and mechanical waves are introduced from the top of the breast. $\mathbf{f}$ Two mechanical wave transmitters are positioned obliquely in the upper and lower part of the breast that simultaneously produces mechanical waves [36]

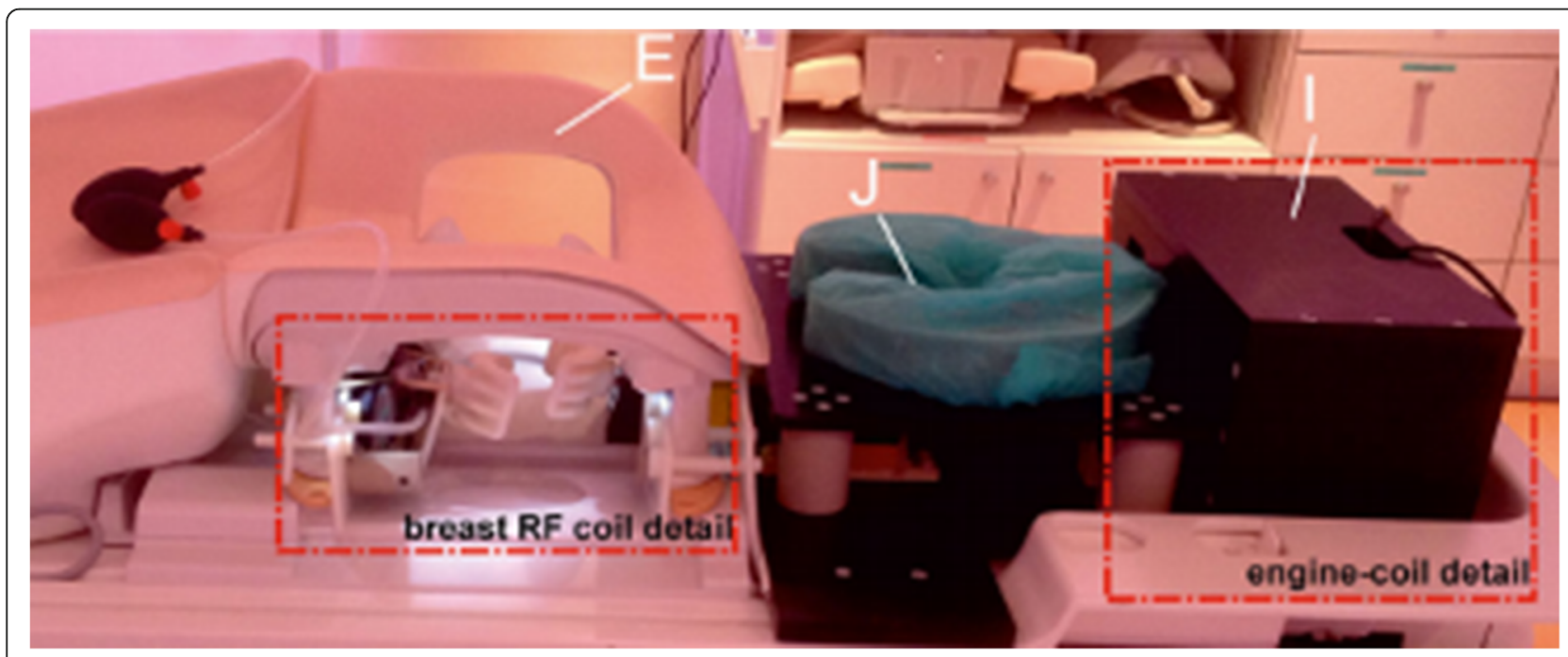

Fig. 15 An overview of MRE equipment including RF coil (left) and engine coil (right) [36] 


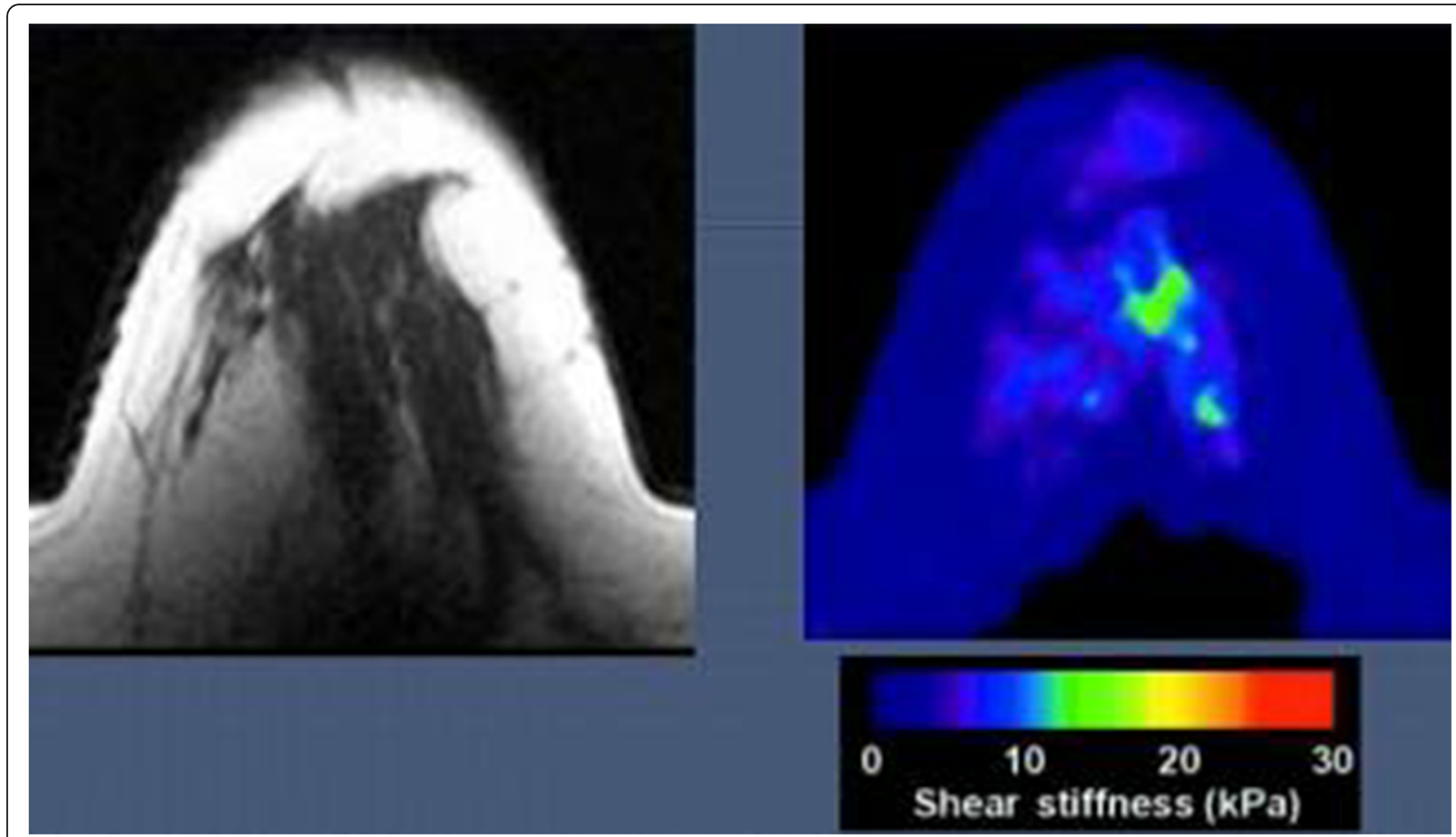

Fig. $16 T_{1}$ image shows healthy breast tissue MRI (left), while MRI elastography shows a heterogeneous complication (right). Permission was acquired to display the images from Dr. Inas M. Elfiki

breast lesions. The information obtained from this method is used for a variety of applications including monitoring the response to cancer treatments and improving the accuracy of lesion diagnosis. The first study on MRS was the measurement of phosphorus atoms' (31P) resonance of the breast. These studies have shown that measurable changes in phospholipid metabolism can be used to detect cancer and monitor response to treatment. Currently, hydrogen atoms (1H-MRS) are used in MRS because of their high sensitivity compared to phosphorus. Initial reports indicated the basis of $1 \mathrm{H}$ MRS in water and fat ratios in the breast. However, later studies showed that this ratio was not found to be a useful predictor. Several studies with $1 \mathrm{H}$-MRS have shown that total choline-induced resonances (tCho) are commonly present in malignant lesions that are not seen in benign or normal tissues. Numerous in vivo, ex vivo, and in vitro studies have shown that this tCho peak is increased in neoplastic tissues. The first and most applicable feature of breast MRS is the diagnosis of malignant from benign lesions prior to biopsy. tCho can be used as a marker of malignancy. The sensitivity of this method is $83 \%$ and its specificity is reported to be $85 \%$, which has very promising results. A second and perhaps more promising use of breast MRS is to predict response to cancer treatment. Existing clinical methods such as breast examination and palpation and tumor-size imaging require several weeks to produce detectable changes. Whereas, breast MRS reveals intracellular metabolism changes prior to any morphological changes [37]. In general, MRS is used to assess the level of tissue metabolism in the body. Its equipment is similar to a conventional MRI with sequences for gaining spectral signals to measure the distribution of specific metabolism in a volume [14] (Fig. 17).

\section{Nuclear medicine imaging}

Nuclear medicine is used in oncology imaging to diagnose, plan, and respond to treatment. In these modalities, specific radiopharmaceuticals are injected intravenously and images that indicate drug uptake in organs are collected in scintillation [14]. Generally, nuclear breast imaging is performed in two ways: single-photon emission computed tomography (SPECT) and positron emission tomography of breast (PET). SPECT is a physiological imaging method while PET is a metabolic and molecular imaging modality [38]. In SPECT, after injection of ${ }^{99 \mathrm{~m}} \mathrm{Tc}$, it is uptaked in activated mitochondria in malignant breast cells. Emitted gammas with $140 \mathrm{keV}$ energy, collected in a large planar gamma detector that lies beneath the compressed breast [18]. The SPECT sensitivity for small lesions is low due to the low spatial resolution. Furthermore, the radiation dose in SPECT is approximately 20-30 times higher than digital mammography [14]. In SPECT study, sensitivity 


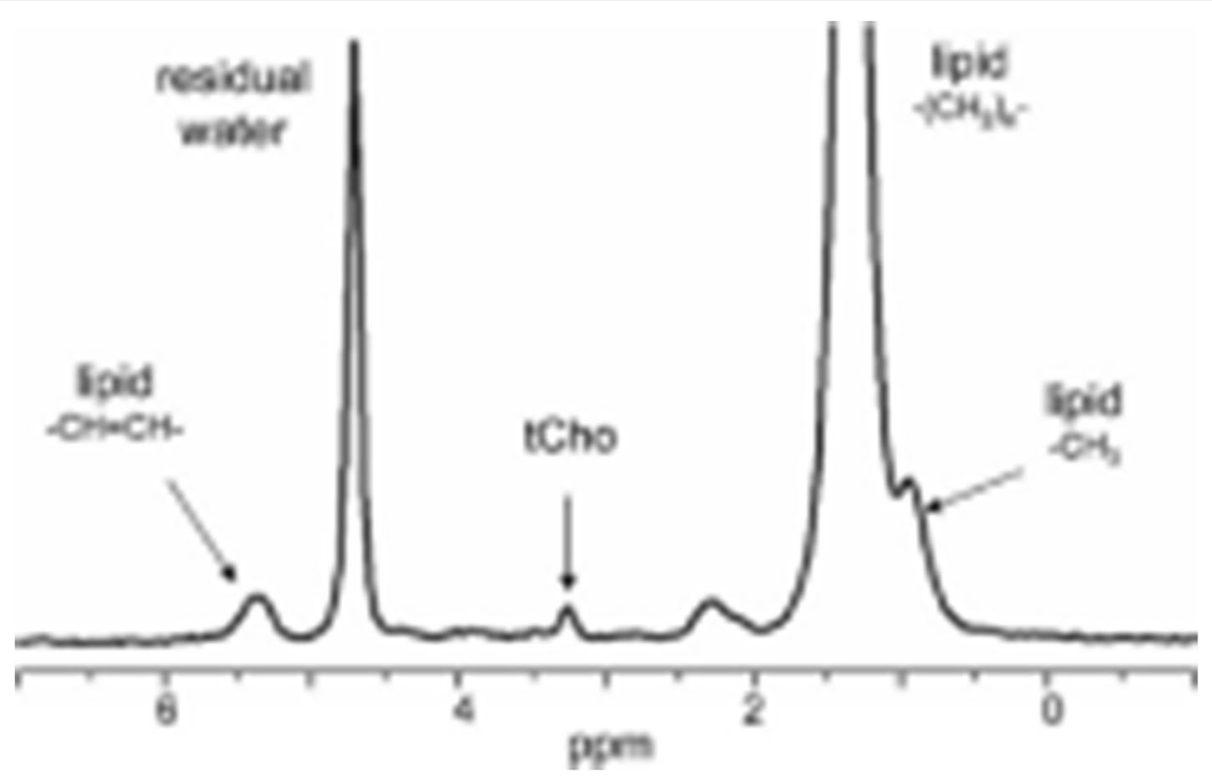

Fig. 17 tCho peak is an indicator of a malignant lesion in breast tissu e[37]

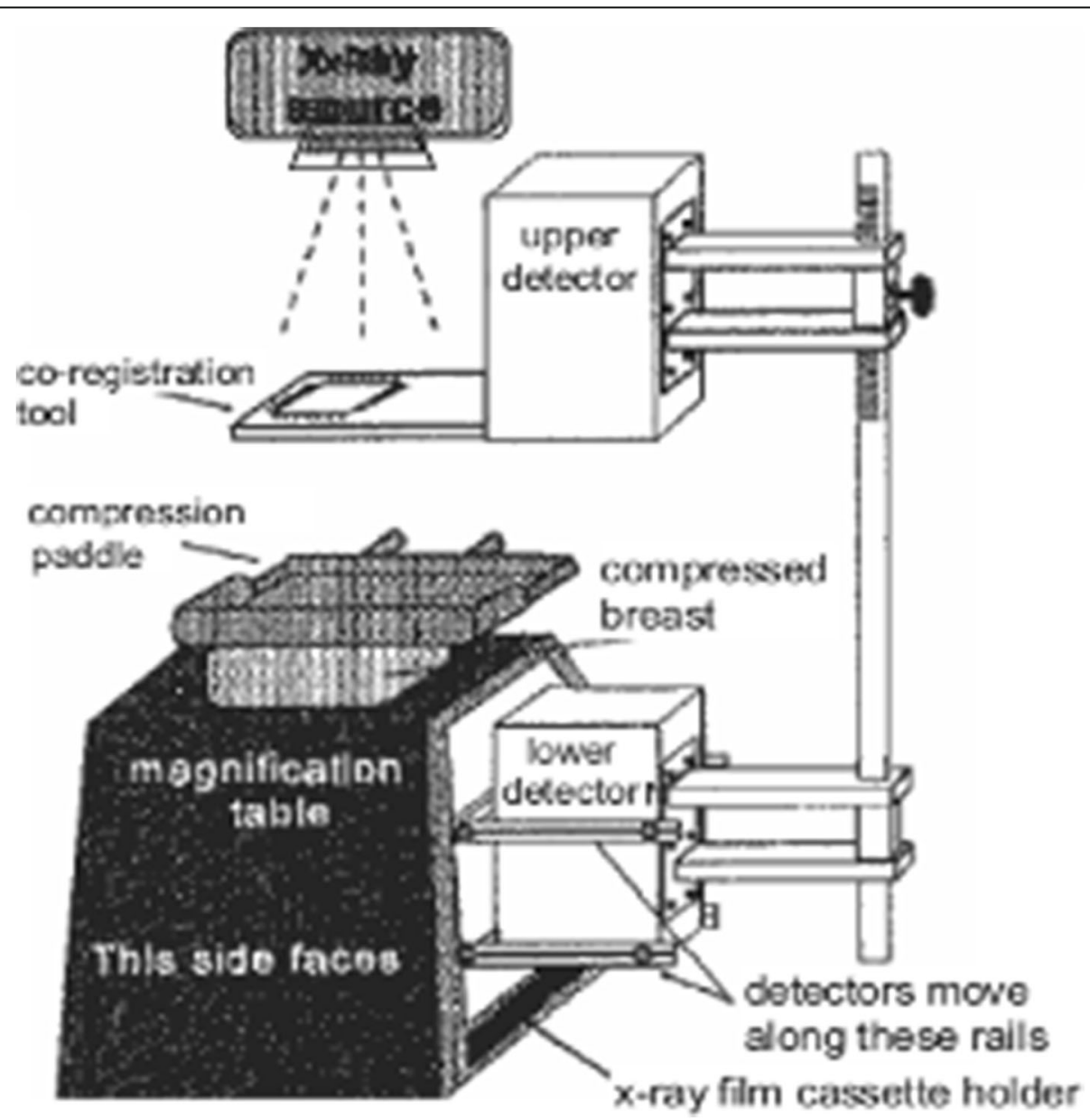

Fig. 18 A view of PEM detectors installed on conventional mammography [42] 
and specificity were resulted in $96.4 \% 59.5 \%$ respectively. Therefore, this method with high sensitivity is a robust examination to detect the exact location of the lesions in a patient who has already been diagnosed with cancer. Moreover, due to low specificity, high false-negative as well as high radiation dose has caused SPECT method hardly used in low-risk cases [39]. Positron emission tomography (PET) on the other hand, is an imaging technique for quantification of the biochemical and physiological activity of cells in a molecular level [38]. PET is often used to identify the grade, stage, and evaluation of treatment response as well. The increased uptake of glucose in malignant cells has been well known for many years, so one of the most widely used PET tracers is the fluorinated analog of glucose, ${ }^{18} \mathrm{~F}$-2-deoxy-D-glucose [14]. ${ }^{18} \mathrm{~F}$ of FDG is a positron-emitting radionuclide. Each positron combines with an electron and then produces two $511 \mathrm{keV}$ gamma rays. PET in breast imaging has higher spatial resolution than SPECT and can detect smaller lesions (about 2.2 $\mathrm{mm})$. SPECT radiation dose is about $1.1 \mathrm{mSv}$ less than PEM. Furthermore, PEM is an expensive method as its cost is approximately 3.7 times higher than SPECT [40]. In one procedure, the sensitivity and specificity of PEM method were about $90 \%$ and $86 \%$ respectively [41]. In a brief, SPECT and PET are not very common methods in early detection of low-risk cases because of delivers doses equivalent to $30-40$ mammograms and low specifies as well [18] Figs. 18, 19 and 20.

\section{Optical imaging}

Optical imaging is a broad keyword that covers all imaging with non-ionized visible, ultraviolet, and infrared electromagnetic waves. This is a non-invasive imaging technique that is currently using near-infrared (NIR) wavelengths (700-1000 nm) and visible light (400-700 $\mathrm{nm})$ to investigate molecular, morphological, and functional information of the tissue and also for absorption, scattering, and fluorescence properties. One of the advantages of optical imaging is its ability to obtain information at the metabolic and molecular levels that can produce images of microscopic structures and tissue function $[43,44]$. The advantages of the optical method are its source uses non-ionizing beams of infrared light; it is a low-cost method and provides physiological and molecular images. One of the major obstacles and challenges in optical methods for clinical imaging is the depth of penetration of light into the tissue. Over the past 15 years, extensive research has been conducted on optical breast imaging techniques. NIR light has a high penetration ability in soft tissue. The main features of breast cancer masses are the abnormal growth of the vascular and tissue lymphatic system, disruption of the biochemical and biophysical equilibrium of the extracellular matrix (ECM), and changes in hemoglobin rate $(\mathrm{HbR})$ and oxyhemoglobin $\left(\mathrm{HbO}_{2}\right)$ concentrations considering the fact that each of which can be an optical image contrast contributing factor. Choosing the wavelength of the optical imaging method is very effective in visualizing different tissue components. In order to image the total hemoglobin, a wavelength of $800 \mathrm{~nm}$ should be selected, whereas dioxide hemoglobin is more sensitive at wavelength of $650-690 \mathrm{~nm}$, and for the detection of water and fat, a wavelength of 925-975 nm should be selected. This method in conjunction with absorption information acquired from Mie scattering analysis (Mie scattering is elastic scattered light of particles that have a diameter similar to or larger than the wavelength of the incident light) can give an estimate of the

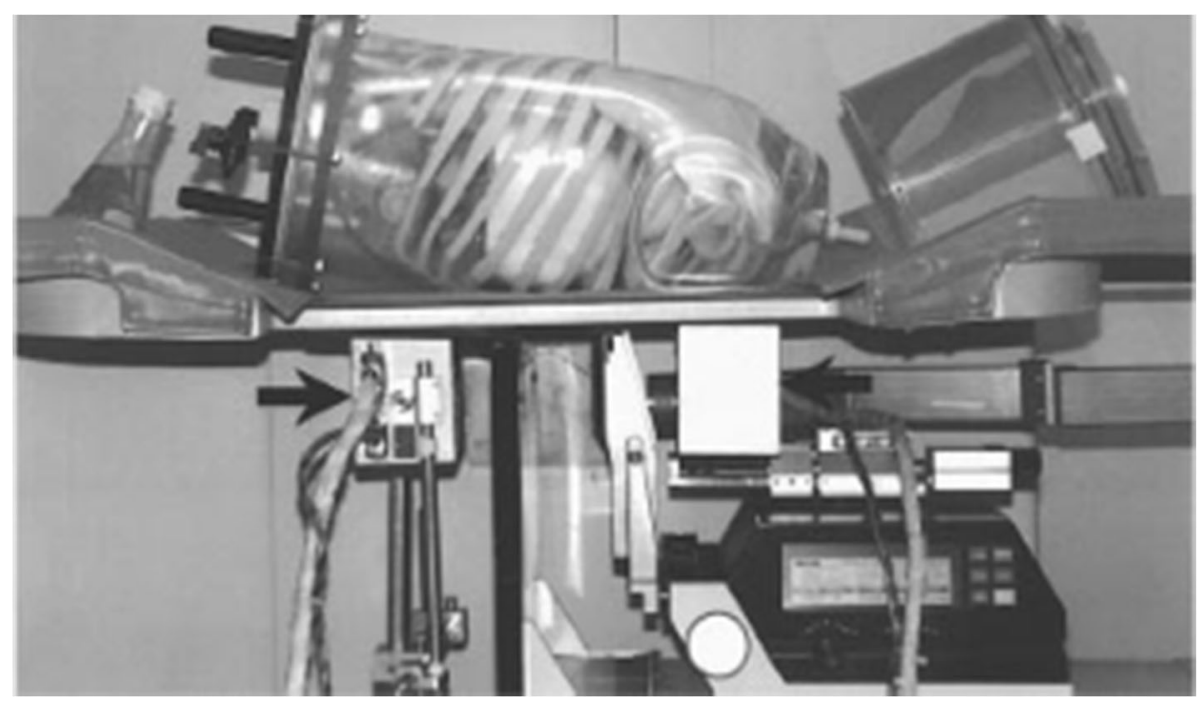

Fig. 19 PEM detectors installed on an autopsy system [42] 


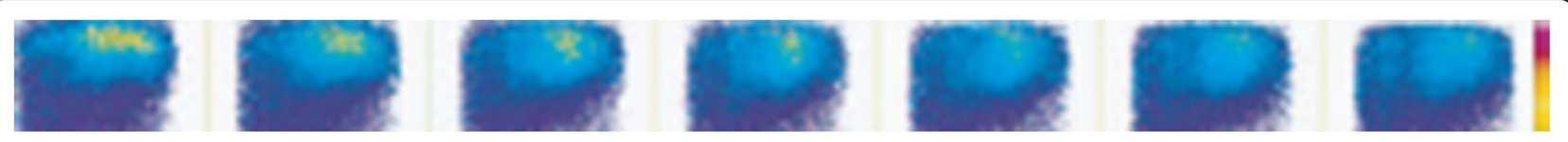

Fig. 20 A collection of images acquired with a PET scanner [42]

size and density of scattering centers [5]. In breast cancer, an increase in hemoglobin concentration and a decrease in oxygen saturation is observed. Although oxygen saturation does not decrease in all breast cancers, it depends on the vascular network and angiogenesis. Structural, vascular, and molecular studies of the breast are feasible with OI which is a cheap, simple, and patient-tolerant technique. However, it is not qualified to diagnose breast cancer because it has less spatial resolution and accuracy. OI can provide significant details about cancer without contrast media injection [45]. According to one study, the sensitivity and specificity of this method was $96 \%$ and 93\%, respectively. Optical imaging is an adjacent device for mammography or ultrasound imaging because the information obtained by the optical signal is distinctly different from that obtained with clinical imaging. NIR scan of suspected malformations may provide additional information needed to better determine malignant conditions and determine if the biopsy is needed and can also detect malformations over time. Hence, the potential is to add optical devices into a secondary imaging modality to provide information that can help diagnose the lesion and follow treatment response [44].

The first generation of optical breast imaging is by compressing the tissue with a transparent two-sided

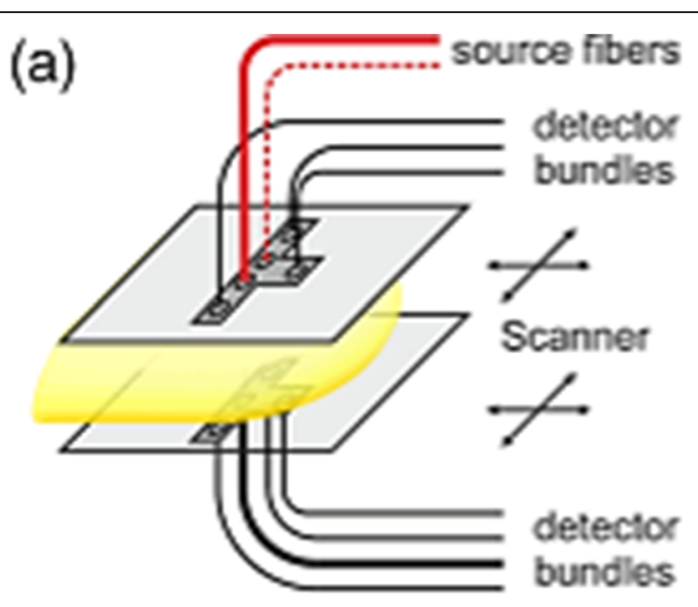

(d)

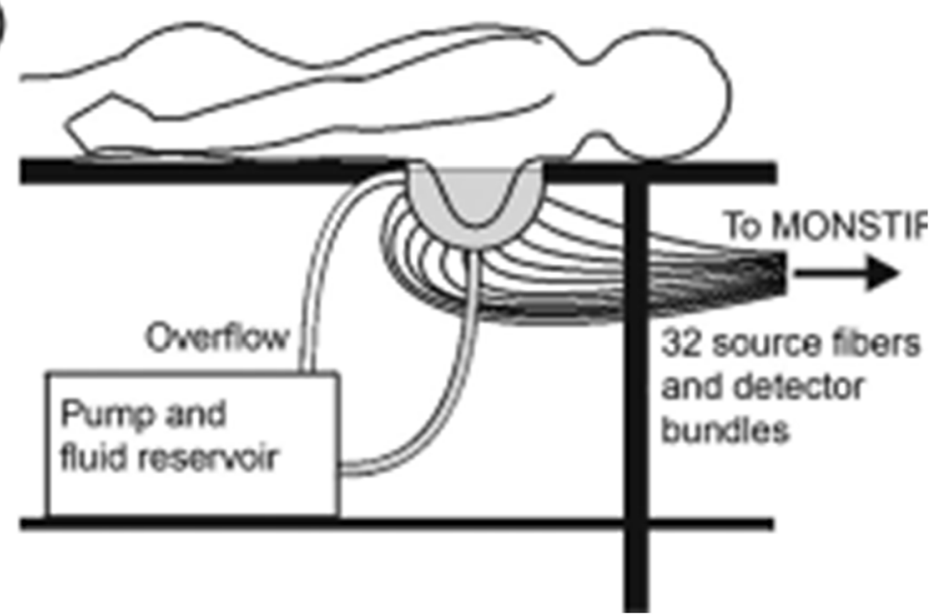

Fig. 21 Optical imaging method with breast tissue compression [5] 
screen and scanning it with a fiber source and a fiber optic detector, known as the parallel plate method. This method was first proposed by Siemens and Carl Zeiss in a frequency-domain type (Fig. 21). The next generation of scanners is a conical chamber (the shape of a hanging breast), which is surrounded by source and detector optical fibers. The feature of this generation is the production of 3D images with the actual size and shape of the breast, which makes it possible to use these images directly for treatment. Philips has designed a continuouswave sample, and the London College has designed Time-Domain type capable of measuring absorption and scattering coefficients (Fig. 22). The third generation of optical breast imaging was unveiled at the University of California. This method is actually a type of molecular spectroscopy (DOIS). This method often determines the blood flow information and the concentration of blood factors. This generation has a hand-held probe with two source fibers and two optical fiber detectors, one of which is a pair of source-detectors operating in the 6wavelength-frequency phase $(650-860 \mathrm{~nm})$, and the second pair of source detectors producing white tungsten light with 650-1000 $\mathrm{nm}$ wavelengths acquiring continuous-wave $(\mathrm{CW})$ type information. At the University of Pennsylvania, a hand-held probe of three sourcedetector pairs was designed and presented in the CW phase (Fig. 23). Depending on the position of the patient in the above three methods, the information of the optical imaging modalities can be combined with the anatomical images of mammography, MRI, or ultrasound. By combining optical imaging with other imaging techniques, the anatomical, metabolic, and physiological information of the tissue can also be obtained. In one study, a manual probe combined ultrasound imaging and optical spectroscopy. Combining the optical method with MRI is a major challenge that optical method electronic systems can affect the RF frequencies of the device and coils unless the fiber length is very high. The first combination of the optical method with MRI was reported by Niziachristos and Ma. In another study at the University of Massachusetts Hospital, a parallel-plate optical method was combined with conventional X-ray mammography [5] Figs 24 and 25.

\section{Breast microwave imaging}

Breast microwave imaging is a cancer detection method in the breast that uses microwave (the range of waves in which wavelength is shorter than radio waves and more than infrared). The wavelength of the microwave is between $1 \mathrm{~mm}$ (corresponding to $300 \mathrm{GHz}$ ) and up to 30 $\mathrm{cm}$ (corresponding to $1 \mathrm{GHz}$ ). This range of wavelength has acceptable penetration in the soft tissue of the breast. This method is used to identify malignant and benign tissues. Although these techniques require a high

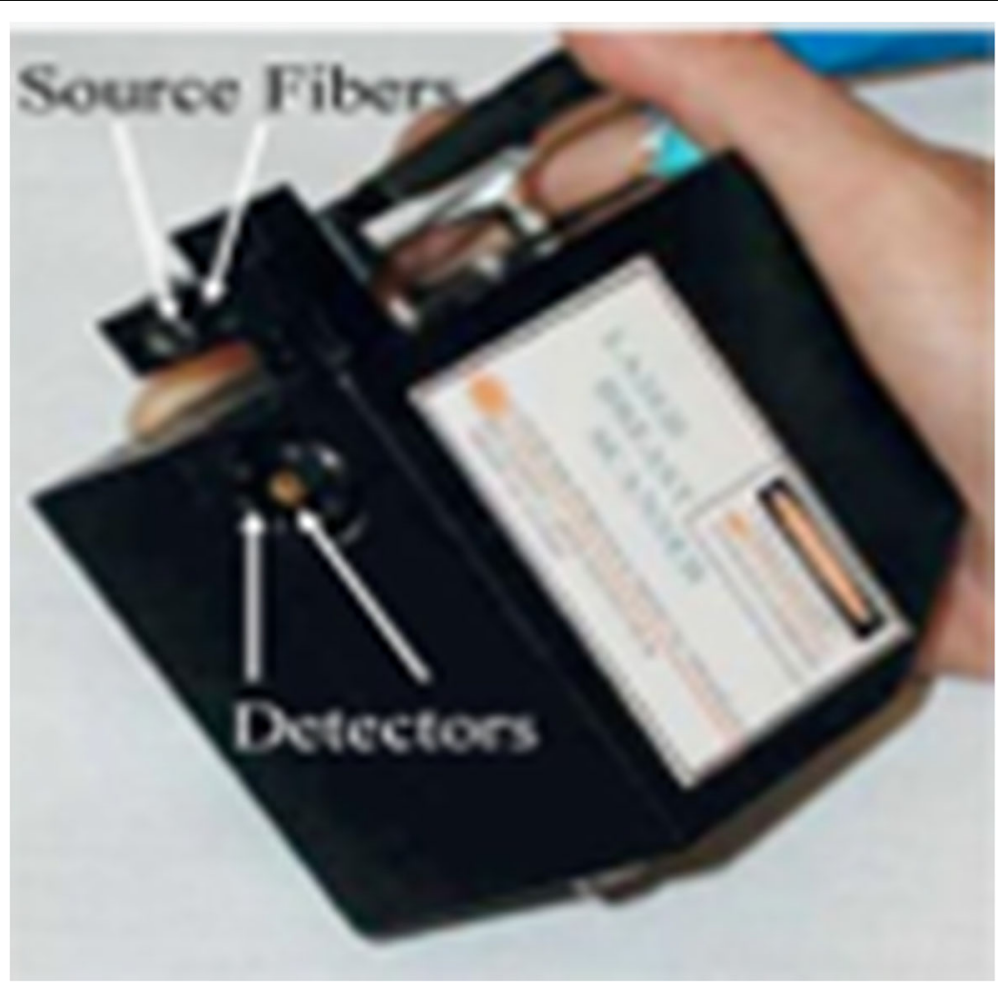

Fig. 22 Optical imaging method with hanging breasts [5] 


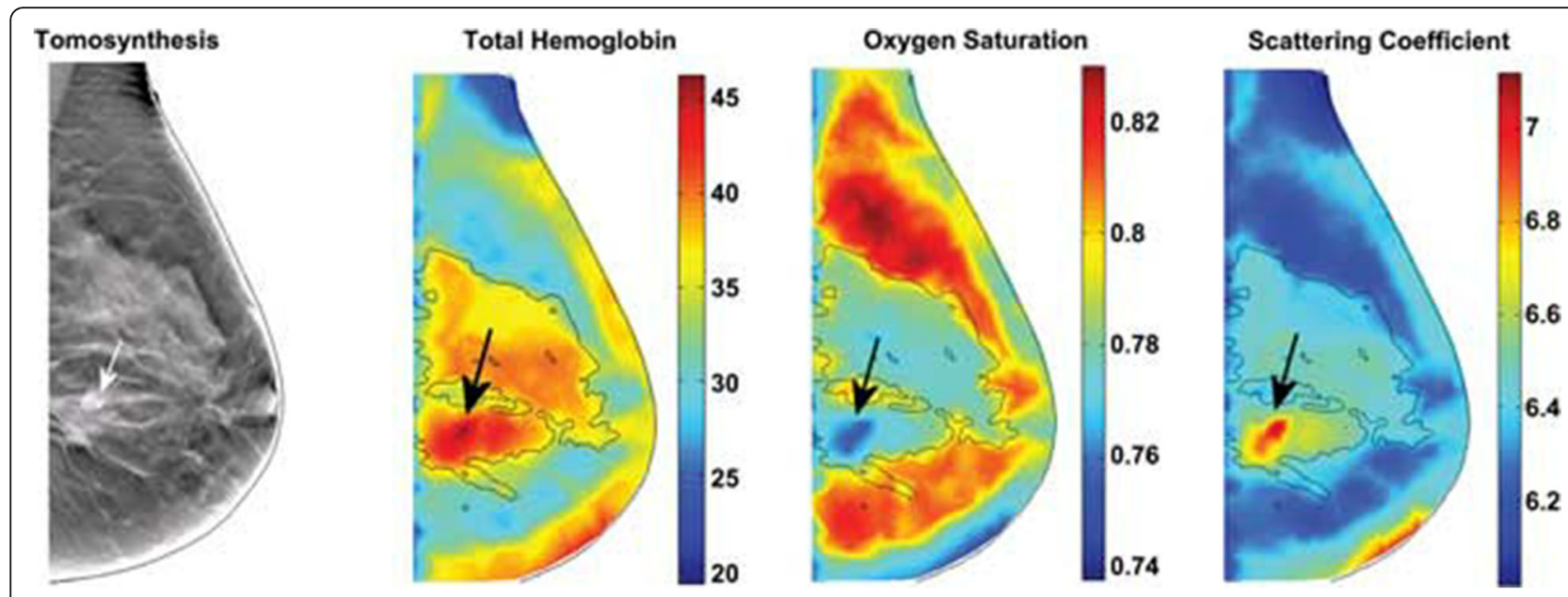

Fig. 23 Optical spectroscopy device probe for the measurement of breast blood factor concentrations [5]

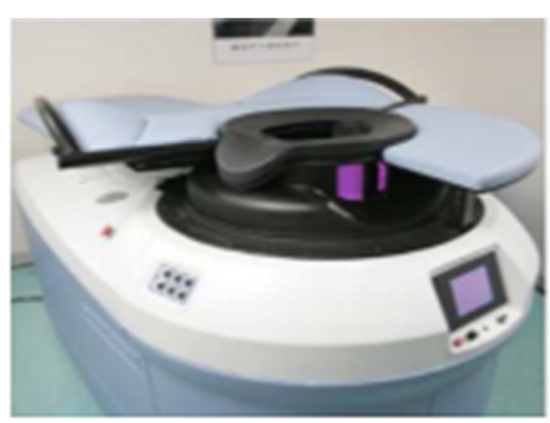

A
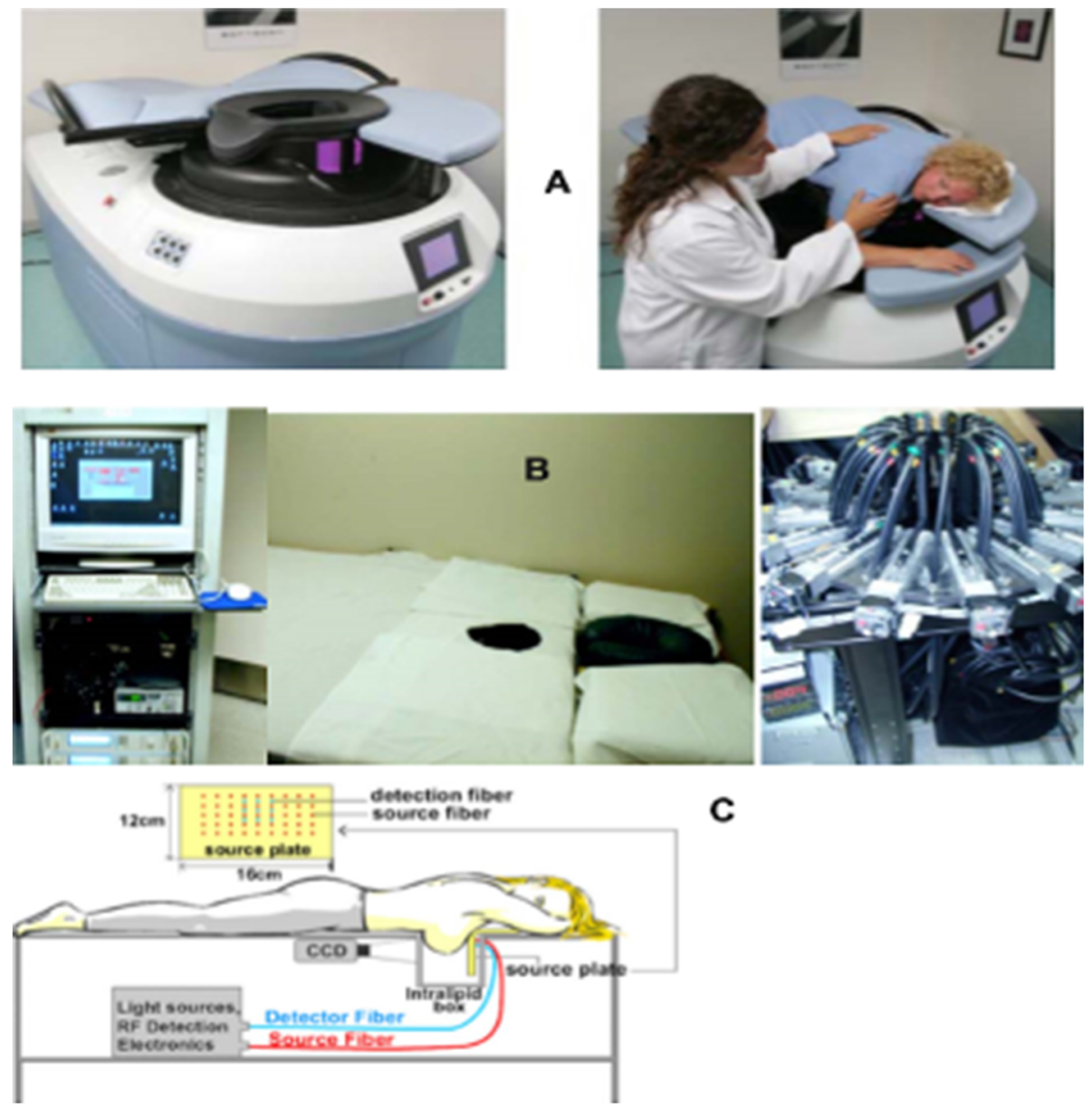

C

Fig. 24 Optical image of breast with anomaly—(in the left to right order) tomosynthesis, overall hemoglobin pattern, oxygen saturation pattern and scatter pattern image. Authorization was acquired to display Qianqian Fang's image [46] 


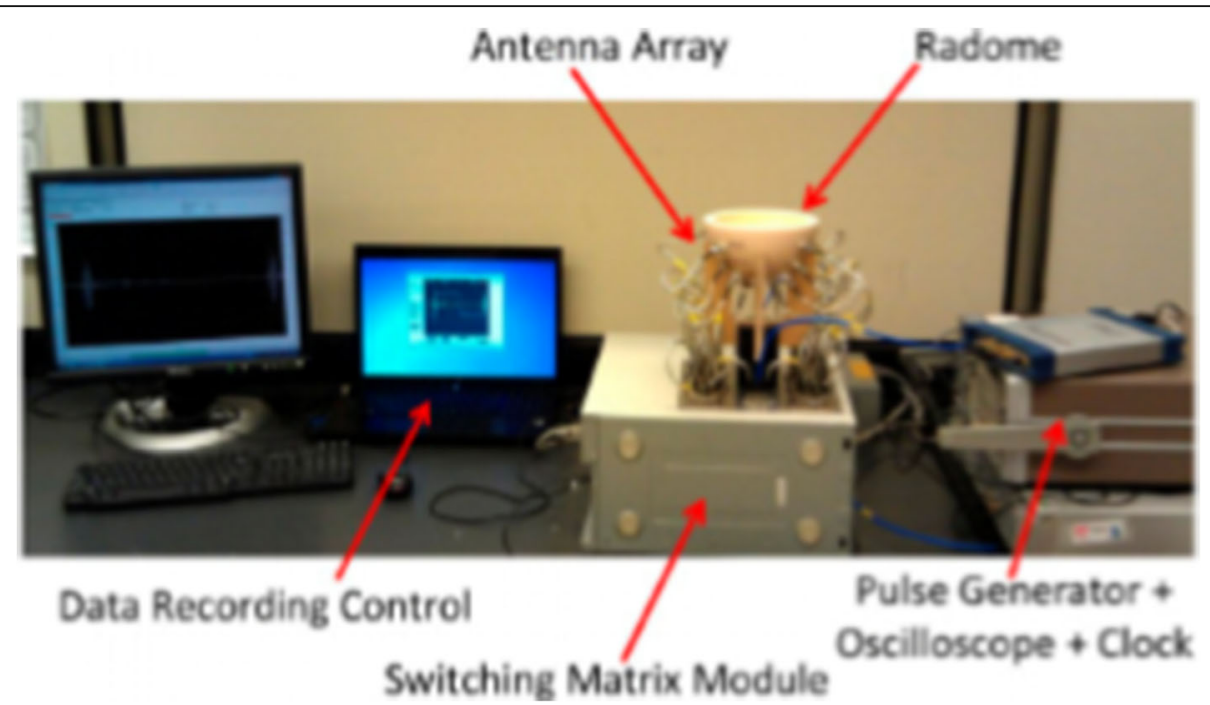

Fig. 25 All of these systems use NIR light to reconstruct images of hemoglobin, oxygen saturation, water, and scattering values through the breast [44]

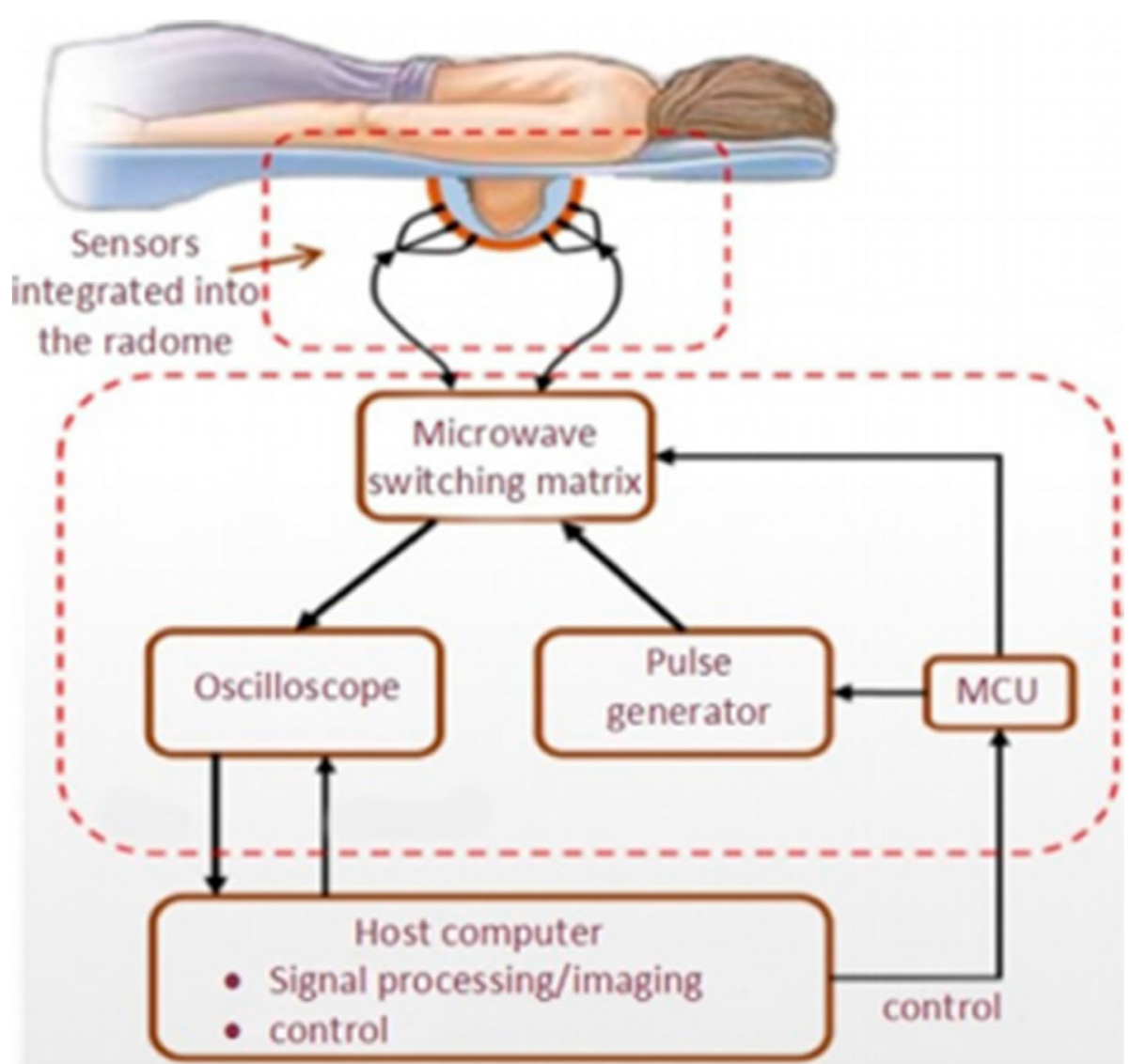

Fig. 26 Breast microwave imaging apparatus (above), positioning (below) [4] 
cost, in recent decades, biosensor-based methods for cancer detection have gained much attention. The quality of the breast microwave image is determined by the microwave sensor, the sensor array, the number of sensors in the array, and the sensor size (Fig. 26) [4].

A biosensor typically comprises cancer target markers, bioreceptors, and biocompatible transducer components. Common features of microwave sensors for detecting breast cancer are wide impedance bandwidth, small size, reproducibility, reasonable cost, and good power pairing with the breast. Among the methods studied for the diagnosis of breast cancer are based on biomarkers immunohistochemistry, radioimmunoassay, fluoroimmunoassay, and immunosorbent acid enzyme linkage. Breast microwave imaging depends on the dielectric properties between healthy tissue and tumor tissue. Different biological tissues containing water have different electrical properties. Microwave imaging is divided into two categories: microwave tomography, which provides little information on the dielectric properties of breast tissue for tumor detection, and radar-based microwave imaging, which is the main type of microwave imaging, maps the internal organ structure by measuring the dielectric properties of the tissues. The reasonable cost, sensitivity, high specificity, and safety of this method are its advantages and is more convenient and reliable than microwave tomography [4].

In this method, the resolution is greatly affected by the shortest wavelength of microwave radiation; therefore, the resolution is related to the frequency range of the microwave radiation. In general, the shorter the wavelength, the more details the image achieves, which implies better resolution. In microwave imaging, a shorter wavelength means less penetration depth. The diagnosis of breast tumors requires a resolution below centimeterscale, which is available with a frequency of about 10 $\mathrm{GHz}[18]$.

\section{Conclusion}

Currently, breast cancer has become one of the health challenges in human societies. Early detection of breast cancer plays an important role in its treatment and control. If breast cancer is diagnosed early, it is known to have a very high survival rate. There are various methods for screening and diagnosing breast cancer including mammography, ultrasound, magnetic resonance imaging, nuclear medicine imaging, optical imaging, and breast microwave imaging. Each of these methods has different subdivisions and their advantages and disadvantages have been discussed. Although there are ways to improve these methods, it can be kept in mind that with the simultaneous use of the capabilities of each imaging technique and their combination, early detection of breast cancer can be improved.

\section{Abbreviations}

MLO: Mediolateral oblique views; CC: Craniocaudal view; DBT: Digital breast tomosynthesis; CEDM: Contrast-enhanced digital mammography; ABUS: Automatic breast ultrasound; CEUS: Contrast-enhanced ultrasound; SWE: Shear wave elastography; MRI: Magnetic resonance imaging; DWI: Diffusion-weighted imaging; MRE: Magnetic resonance elastography; MRS: Magnetic resonance spectroscopy; PEM: Positron emission mammography; 18F-FDG: Fluorodeoxyglucose; NIR: Near infrared; $\mathrm{HbR}$ : Hemoglobin rate; $\mathrm{HbO}_{2}$ : Oxyhemoglobin; $\mathrm{CW}$ : Continuous wave; tCho: Total choline-induced resonances

\section{Acknowledgements}

Not applicable

\section{Authors' contributions}

SI: literature search, data collection, manuscript drafting. TM: study design, data interpretation, revised the manuscript. FS: data interpretation, revised the manuscript. MFG: data collection, data interpretation, revised the manuscript. AG: data collection, manuscript drafting. DKh: study design, data interpretation, revised the manuscript. AEM: revised the manuscript. All authors have read and approved the final manuscript.

\section{Funding}

Not applicable

Availability of data and materials

Not applicable

Ethics approval and consent to participate

Not applicable

Consent for publication

Not applicable

\section{Competing interests}

The authors declare that they have no competing interests.

\section{Author details}

${ }^{1}$ Department of Radiology, Paramedical School, Tabriz University of Medical Sciences, Tabriz, Iran. ${ }^{2}$ Department of Medical Physics, School of Medicine, Tabriz University of Medical Sciences, Tabriz, Iran. ${ }^{3}$ Department of Medical Physics, Tehran University of Medical Sciences, Tehran, Iran.

Received: 11 January 2020 Accepted: 19 March 2020

Published online: 16 April 2020

\section{References}

1. Harbeck N, Gnant M (2017) Breast cancer. The Lancet 389 (10074):11341150. doi:https://doi.org/https://doi.org/10.1016/S0140-6736(16)31891-8.

2. Bray F, Ferlay J, Soerjomataram I, Siegel RL, Torre LA, Jemal A (2018) Global cancer statistics 2018: GLOBOCAN estimates of incidence and mortality worldwide for 36 cancers in 185 countries. CA Cancer J Clin 68(6):394-424

3. Ghoncheh M, Pournamdar Z, Salehiniya H (2016) Incidence and mortality and epidemiology of breast cancer in the world. Asian Pac J Cancer Prev 17(S3):43-46

4. Wang $L$ (2018) Microwave sensors for breast cancer detection. Sensors 18(2):655

5. Grosenick D, Rinneberg H, Cubeddu R, Taroni P (2016) Review of optical breast imaging and spectroscopy. J Biomed Opt 21(9):091311

6. Jemal A, Bray F, Center MM, Ferlay J, Ward E, Forman D (2011) Global cancer statistics. CA Cancer J Clin 61(2):69-90

7. Jordan V, Khan M, Prill D (2019) Breast cancer screening: why can't everyone agree? Prim Care 46(1):97-115

8. Nikolova NK (2011) Microwave imaging for breast cancer. IEEE Microw Mag 12(7):78-94

9. Autier P, Boniol M (2018) Mammography screening: a major issue in medicine. Eur J Cancer 90:34-62

10. Morrow M, Waters J, Morris E (2011) MRI for breast cancer screening, diagnosis, and treatment. Lancet 378(9805):1804-1811

11. Chae EY, Kim HH, Cha JH, Shin HJ, Kim H (2013) Evaluation of screening whole-breast sonography as a supplemental tool in conjunction with 
mammography in women with dense breasts. J Ultrasound Med 32(9): 1573-1578

12. Taori K, Dhakate S, Rathod J, Hatgaonkar A, Disawal A, Wavare P, Bakare V, Puri RP (2013) Evaluation of breast masses using mammography and sonography as first line investigations. Open J Med Imaging 3(01):40

13. Gilbert FJ, Pinker-Domenig K (2019) Diagnosis and Staging of Breast Cancer: When and How to Use Mammography, Tomosynthesis, Ultrasound, Contrast-Enhanced Mammography, and Magnetic Resonance Imaging. In: Hodler J., Kubik-Huch R., von Schulthess G. (eds) Diseases of the Chest, Breast, Heart and Vessels 2019-2022. IDKD Springer Series. Springer, Cham

14. Mortezazadeh T, Gholibegloo E, Riyahi Alam N, Haghgoo S, Musa AE, Khoobi M (2020) Glucosamine conjugated gadolinium (III) oxide nanoparticles as a novel targeted contrast agent for cancer diagnosis in MRI. J Biomed Phys Eng 10(1):25-38. https://doi.org/10.31661/jbpe.v0i0.1018

15. Greenwood HI, Dodelzon K, Katzen JT (2018) Impact of advancing technology on diagnosis and treatment of breast cancer. Surg Clin 98(4): 703-724

16. National Health Council (2006). Health risks from exposure to low levels of ionizing radiation: BEIR VII phase 2 Chapter 12 P267-312

17. Friedewald SM (2018) Breast Cancer Screening: The Debate that Never Ends. In: Gradishar W. (eds) Optimizing Breast Cancer Management. Cancer Treatment and Research, vol 173. Springer, Cham

18. Drukteinis JS, Mooney BP, Flowers Cl, Gatenby RA (2013) Beyond mammography: new frontiers in breast cancer screening. Am J Med 126(6): 472-479

19. Dromain C, Balleyguier C (2010) Contrast-Enhanced Digital Mammography In: Bick U., Diekmann F. (eds) Digital Mammography. Medical Radiology. Springer, Berlin, Heidelberg

20. Jochelson M (2014) Contrast-enhanced digital mammography. Radiol Clin 52(3):609-616

21. Blum K, Antoch G, Mohrmann S, Obenauer S (2015) Use of low-energy contrast-enhanced spectral mammography (CESM) as diagnostic mammography-proof of concept. Radiography 21(4):352-358

22. Badr S, Laurent N, Régis C, Boulanger L, Lemaille S, Poncelet E (2014) Dualenergy contrast-enhanced digital mammography in routine clinical practice in 2013. Diagn Interv Imaging 95(3):245-258

23. Diekmann F, Freyer M, Diekmann S, Fallenberg EM, Fischer T, Bick U, Pöllinger A (2011) Evaluation of contrast-enhanced digital mammography. Eur J Radiol 78(1):112-121

24. Hedrick WR, Hykes DL, Starchman DE (2005) Ultrasound physics and instrumentation: practice examinations. Fourth Edition. CV Mosby. Chapter 5 . P65-82

25. Guo R, Lu G, Qin B, Fei B (2018) Ultrasound imaging technologies for breast cancer detection and management: A review. Ultrasound Med Biol 44(1): 37-70

26. Brem RF, Lenihan MJ, Lieberman J, Torrente J (2015) Screening breast ultrasound: past, present, and future. AJR Am J Roentgenol 204(2):234-240

27. Carpentier B, Hayward J, Strachowski L (2017) Enhancing your acoustics: ultrasound image optimization of breast lesions. J Ultrasound Med 36(7): 1479-1485

28. Kim HJ, Kim SM, Kim B, La Yun B, Jang M, Ko Y, Lee SH, Jeong H, Chang JM, Cho N (2018) Comparison of strain and shear wave elastography for qualitative and quantitative assessment of breast masses in the same population. Sci Rep 8(1):6197

29. Regini E, Bagnera S, Tota D, Campanino P, Luparia A, Barisone F, Durando M, Mariscotti G, Gandini G (2010) Role of sonoelastography in characterising breast nodules. Preliminary experience with 120 lesions. La Ons. Radiol Med 115 (4):551-562

30. Barr RG (2018) The Role of Sonoelastography in Breast Lesions. Seminars in Ultrasound, CT and MRI 39(1):98-105

31. Gennisson J-L, Deffieux T, Fink M, Tanter M (2013) Ultrasound elastography: principles and techniques. Diagn Interv Imaging 94(5):487-495

32. Zhang Y, Ren H (2017) Meta-analysis of diagnostic accuracy of magnetic resonance imaging and mammography for breast cancer. J Cancer Res Ther 13(5):862

33. Maxwell A, Lim Y, Hurley E, Evans D, Howell A, Gadde S (2017) Falsenegative MRI breast screening in high-risk women. Clin Radiol 72(3):207-216

34. Phillips J, Miller MM, Mehta TS, Fein-Zachary V, Nathanson A, Hori W, Monahan-Earley R, Slanetz PJ (2017) Contrast-enhanced spectral mammography (CESM) versus MRI in the high-risk screening setting: patient preferences and attitudes. Clin Imaging 42:193-197
35. Partridge SC, Nissan N, Rahbar H, Kitsch AE, Sigmund EE (2017) Diffusionweighted breast MRl: clinical applications and emerging techniques. hniques. J Magn Reson Imaging 45(2):337-355

36. Bohte A, Nelissen J, Runge J, Holub O, Lambert S, de Graaf L, Kolkman S, Van Der Meij S, Stoker J, Strijkers G (2018) Breast magnetic resonance elastography: a review of clinical work and future perspectives. NMR Biomed 31(10):e3932

37. Bolan PJ, Nelson MT, Yee D, Garwood M (2005) Imaging in breast cancer: magnetic resonance spectroscopy. Breast Cancer Res 7(4):149

38. Groheux D, Cochet A, Humbert O, Alberini J-L, Hindié E, Mankoff D (2016) 18F-FDG PET/CT for staging and restaging of breast cancer. J Nucl Med 57(Suppl 1):17S-26S

39. Brem RF, Floerke AC, Rapelyea JA, Teal C, Kelly T, Mathur V (2008) Breastspecific gamma imaging as an adjunct imaging modality for the diagnosis of breast cancer. Radiology 247(3):651-657

40. Yamamoto Y, Tasaki Y, Kuwada Y, Ozawa Y, Inoue T (2016) A preliminary report of breast cancer screening by positron emission mammography. Ann Nucl Med 30(2):130-137

41. Marino MA, Helbich TH, Blandino A, Pinker K (2015) The role of positron emission tomography in breast cancer: a short review. memo-Magazine of. Eur Med Oncol 8(2):130-135

42. Martins MV (2015) Positron emission mammography. Mammography Techniques and Review:39.

43. Godavarty A, Rodriquez S, Jung Y-J, Gonzalez S (2015) Optical imaging for breast cancer prescreening. Breast Cancer (Dove Med Press) 7:193

44. Tromberg BJ, Pogue BW, Paulsen KD, Yodh AG, Boas DA, Cerussi AE (2008) Assessing the future of diffuse optical imaging technologies for breast cancer management. Med Phys 35 (6Part1):2443-2451.

45. Leo GD, Trimboli RM, Sella T, Sardanelli F (2017) Optical imaging of the breast: basic principles and clinical applications. AJR Am J Roentgenol 209(1):230-238

46. Fang Q, Selb J, Carp SA, Boverman G, Miller EL, Brooks DH, Moore RH, Kopans DB, Boas DA (2011) Combined optical and X-ray tomosynthesis breast imaging. Radiology 258(1):89-97

\section{Publisher's Note}

Springer Nature remains neutral with regard to jurisdictional claims in published maps and institutional affiliations.

\section{Submit your manuscript to a SpringerOpen ${ }^{\circ}$ journal and benefit from:}

- Convenient online submission

- Rigorous peer review

- Open access: articles freely available online

- High visibility within the field

- Retaining the copyright to your article

Submit your next manuscript at $\boldsymbol{\nabla}$ springeropen.com 